\title{
Bypass to Turbulence in Hydrodynamic Accretion Disks: An Eigenvalue Approach
}

\section{Citation}

Mukhopadhyay, Banibrata, Niayesh Afshordi, and Ramesh Narayan. 2005. "Bypass to Turbulence in Hydrodynamic Accretion Disks: An Eigenvalue Approach." The Astrophysical Journal 629 (1): 383-96. https://doi.org/10.1086/431419.

\section{Permanent link}

http://nrs.harvard.edu/urn-3:HUL.InstRepos:41384962

\section{Terms of Use}

This article was downloaded from Harvard University's DASH repository, and is made available under the terms and conditions applicable to Other Posted Material, as set forth at http:// nrs.harvard.edu/urn-3:HUL.InstRepos:dash.current.terms-of-use\#LAA

\section{Share Your Story}

The Harvard community has made this article openly available.

Please share how this access benefits you. Submit a story.

Accessibility 


\title{
Bypass to Turbulence in Hydrodynamic Accretion Disks: An Eigenvalue Approach
}

\author{
Banibrata Mukhopadhyay ${ }^{1}$, Niayesh Afshordi ${ }^{2}$, Ramesh Narayan ${ }^{3}$ \\ Institute for Theory and Computation, Harvard-Smithsonian Center for Astrophysics, 60 Garden \\ Street, MS-51, Cambridge, MA 02138
}

\begin{abstract}
Cold accretion disks with temperatures below $\sim 3000 \mathrm{~K}$ are likely to be composed of highly neutral gas. The magnetorotational instability may cease to operate in such disks, so it is of interest to consider purely hydrodynamic mechanisms of generating turbulence and angular momentum transport. With this motivation, we investigate the growth of hydrodynamic perturbations in a linear shear flow sandwiched between two parallel walls. The unperturbed flow is similar to plane Couette flow but with a Coriolis force included. Although there are no exponentially growing eigenmodes in this system, nevertheless, because of the non-normal nature of the eigenmodes, it is possible to have a large transient growth in the energy of perturbations. For a constant angular momentum disk, we find that the perturbation with maximum growth is axisymmetric with vertical structure. The energy grows by more than a factor of 100 for a Reynolds number $R=300$ and more than a factor of 1000 for $R=1000$. Turbulence can be easily excited in such a disk, as found in previous numerical simulations. For a Keplerian disk, on the other hand, similar perturbations with vertical structure grow by no more than a factor of 4 , explaining why the same simulations did not find turbulence in this system. However, certain other two-dimensional perturbations with no vertical structure do exhibit modest growth. For the optimum two-dimensional perturbation, the energy grows by a factor of $\sim 100$ for $R \sim 10^{4.5}$ and by a factor of 1000 for $R \sim 10^{6}$. Such large Reynolds numbers are hard to achieve in numerical simulations and so the nonlinear development of these kinds of perturbations are only beginning to be investigated. It is conceivable that these nearly two-dimensional disturbances might lead to self-sustained three-dimensional turbulence, though this remains
\end{abstract}

\footnotetext{
${ }^{1}$ bmukhopa@cfa.harvard.edu

${ }^{2}$ nafshord@cfa.harvard.edu

${ }^{3}$ rnarayan@cfa.harvard.edu
} 
to be demonstrated. The Reynolds numbers of cold astrophysical disks are much larger even than $10^{6}$; therefore, hydrodynamic turbulence may be possible in disks through transient growth.

Subject headings: accretion, accretion disk — hydrodynamics — turbulence — instabilities

\section{Introduction}

The origin of hydrodynamic turbulence is not fully understood. Many efforts have been devoted to this problem, beginning with the early work of Kelvin, Rayleigh and Reynolds toward the end of the nineteenth century. However, despite a large number of investigations over the decades, the key physics is still poorly understood. One of the reasons for this is that there is a significant mismatch between the predictions of linear stability theory and experimental data. For example, plane Poiseuille flow is known to become turbulent in the laboratory at a Reynolds number $R \sim 1000$, whereas theory predicts that the flow is linearly stable up to $R=5772$. An even more severe discrepancy, one that is of direct interest to astrophysics, occurs in the case of plane Couette flow. Laboratory experiments and numerical simulations show that this flow can become turbulent for $R$ as small as $\sim 350$. However, theoretical analysis shows that the flow is linearly stable for all $R$ up to infinity. Such a large discrepancy indicates that linear stability analysis, based on eigenspectra, is not the best tool for understanding the onset of turbulence. In this paper, we pursue a different approach, the so-called bypass mechanism to transition, which has been popular in the fluid mechanics literature (e.g., Farrell 1988; Butler \& Farrell 1992;

Reddy \& Henningson 1993; Trefethen et al. 1993). We use this approach to study a possible route to turbulence in astrophysical disks.

Accretion disks in astrophysics operate by transferring angular momentum outward by an effective "viscosity." Microscopic molecular viscosity is completely negligible, so it was recognized more than three decades ago (Shakura \& Sunyaev 1973; Lynden-Bell \& Pringle 1974) that angular momentum transfer must occur via turbulence of some sort. However, the physical origin of the turbulence was not identified until the important work of Balbus \& Hawley (1991) who identified the Magneto-Rotational-Instability (MRI) (originally discovered by Velikhov 1959; Chandrasekhar 1960) and showed that this linear instability will operate in the presence of very weak magnetic fields and will lead to magnetohydrodynamic (MHD) turbulence. The MRI is now accepted as the origin of turbulence in most accretion disks. Hawley, Gammie \& Balbus (1995) showed that the MRI 
dies out if the Lorentz force is turned off, and Hawley, Gammie \& Balbus (1996) showed that the magnetic field dies out when the Coriolis force is turned off while retaining the Lorentz forces. In both of these situations, MHD turbulence is absent. In two subsequent papers, Balbus, Hawley \& Stone (1996) and Hawley, Balbus \& Winters (1999) showed through numerical simulations that, whereas pure hydrodynamic turbulence is easily triggered in plane Couette flow (which was already known) and in a constant angular momentum disk, turbulence does not develop in an unmagnetized Keplerian disk even in the presence of large initial perturbations. The authors argued on this basis that hydrodynamic turbulence cannot contribute to viscosity in accretion disks.

Despite the above important work, there is reason to study hydrodynamic turbulence in astrophysical disks. Several accretion systems are known in which the gas is cold and largely neutral so that the gas and the magnetic field are poorly coupled. The MRI then becomes weak or may even cease to operate. In a series of experiments, Hawley, Gammie \& Balbus (1996) and Fleming, Stone \& Hawley (2000) showed that for a magnetic Reynolds number, $R_{M} \sim 10^{4}$, the magnetic field is depressed and for $R_{M}=2 \times 10^{3}$ the magnetic field dies out. Thus, for $R_{M} \leq 10^{4}$, MHD turbulence and the associated angular momentum transport switch off. Examples of systems in this regime include accretion disks around quiescent cataclysmic variables (Gammie \& Menou 1998; Menou 2000), proto-planetary and star-forming disks (Blaes \& Balbus 1994; Gammie 1996; Fromang, Terquem \& Balbus 2002), and the outer regions of disks in active galactic nuclei (Menou \& Quataert 2001; Goodman 2003). Note that the magnetic Reynolds number may not be the only relevant parameter that determines the strength of the MRI; the magnetic Prandtl number may play a role (Gammie \& Menou 1998), as well as ambipolar diffusion (Blaes \& Balbus 1994; Menou \& Quataert 2001). Nevertheless, it seems reasonable to assume that, in cold astrophysical disks, the MRI will be sluggish or even absent. The question then arises: How can these systems sustain mass transfer in the absence of the MRI? What drives their turbulence?

A number of ideas have been discussed in the literature in answer to this question. Gammie (1996) argued that the surface layers of cold protostellar disks would be ionized by cosmic rays and that this would enable accretion to proceed via the MRI within these layers. Menou (2000) suggested that angular momentum transport in quiescent cataclysmic variables could be induced by tidal perturbations from the binary companion star and showed that this mechanism could explain the occurrence of longer outburst recurrence times in systems with large binary mass ratios. In the case of the outer disks of active galactic nuclei, Menou \& Quataert (2001) showed that the MRI-stable regions are nearly always gravitationally unstable, so that the latter might drive angular momentum transport. A problem with these ideas is that each is invoked specifically for a particular class of 
systems. While there is nothing in principle wrong with this, one wonders whether there may not be some more general mechanism for generating turbulent transport in MRI-stable astrophysical disks.

Recent laboratory experiments on rotating Couette flow in the narrow gap limit with linearly stable rotational angular velocity profiles (similar to Keplerian disks) seem to indicate that turbulence does manage to develop in such flows (Richard \& Zahn 1999). Longaretti (2002) points out that the absence of turbulence in the simulations by Balbus, Hawley \& Stone (1996) and Hawley, Balbus \& Winters (1999) may be because of their small effective Reynolds number. Also, Bech \& Andersson (1997) have shown that turbulence persists in numerical simulations of sub-critical rotating flows, provided the Reynolds number is very high. Moreover, as already mentioned, it is well known that linear stability is no guarantee that a flow (whether rotating or not) will avoid becoming turbulent (for a detailed discussion see e.g. Swinney \& Gollub 1981; Drazin \& Reid 1983). In fact, since the celebrated work of Orr (1907), it has been known that linearly stable flows can exhibit significant transient growth in energy for certain initial perturbations. This fact provides a possible solution to the problem of explaining hydrodynamic turbulence in linearly stable systems. The idea is that the transient growth may allow perturbations to grow to a non-linear state, after which a sub-critical transition to turbulence may take place. This is called the bypass mechanism to turbulence. In the astrophysical literature, an early application of transient growth may be found in Goldreich \& Lynden-Bell (1965; see also Goldreich \& Tremaine 1978, 1979).

The physics of transient growth has been discussed by a number of authors (Farrell 1988; Butler \& Farrell 1992; Reddy \& Henningson 1993; Trefethen et al. 1993), who have shown that the growth results from the non-normal nature of the associated operator. The eigenfunctions of the linearly perturbed system are not orthogonal but are close to linearly dependent in nature, and as a result certain linear combinations of the eigenfunctions that are arranged to nearly cancel initially may develop considerable amplitude at later time when the degree of cancellation is reduced. Therefore, even in the absence of any exponentially growing eigenfunctions, the system is still able to exhibit transient growth. This idea has been discussed in the fluid mechanics literature for a number of years but has only recently been applied to astrophysical accretion disks. Ioannaou \& Kakouris (2001) studied the global behavior of perturbations in an accretion disk, Chagelishvili et al. (2003) analysed a local 2-dimensional patch in a disk using a shearing box approximation and showed that strong growth is possible, and Tevzadze et al. (2003) showed that 3-dimensional perturbations also undergo substantial transient growth, provided the vertical scale remains of the order of the azimuthal scale. Umurhan \& Regev (2004) studied the non-linear development of the Chagelishvili et al. (2003) growing mode and Yecko (2004) 
studied rotating shearing flows between walls. Recently, Johnson \& Gammie (2005a,b) studied the evolution of a plane-wave type perturbation in thin low-ionization disks.

The aim of the present study is to further explore the physics of transient non-normal growth of perturbations in cold accretion disks, with a view to understanding whether such growth could lead to hydrodynamic turbulence. Our aim is to present the analysis in such a manner that even readers from other branch of astrophysics (not familiar with the conventional fluid dynamical approach) will be able to follow and reproduce the results. Along with a companion paper (Afshordi, Mukhopadhyay \& Narayan 2005; hereafter AMN05), we study both Keplerian and constant angular momentum disks, as well as plane Couette flow. Both papers concentrate on identifying the parameter regimes over which a large transient growth in energy is possible and studying the nature of the growing perturbations. While the present paper focuses on an eigenvalue analysis in Eulerian coordinates of flow between walls, AMN05 presents a Lagrangian analysis of an infinite shear flow.

The plan of the paper is as follows. In $\S 2$, we present our basic model, beginning with a description of the equilibrium flow, then discussing the perturbation equations and eigenfunctions, and introducing the concept of transient energy growth. In $\S 3$, we present numerical results obtained using the eigenfunction approach for a variety of flows: plane Couette flow, constant angular momentum disk, Keplerian disk. In $\S 4$, we explain the physics of the numerical results by means of analytical and heuristic arguments. Finally, in $\S 5$, we discuss the implications of the results. In the Appendix, we describe the formalism to compute the transient energy growth.

\section{The Model}

\subsection{Equilibrium Flow}

We consider a small patch of an accreting disk centered on radius $r_{0}$ and viewed in a frame orbiting at the angular velocity $\Omega_{0}$ of the gas at this radius. We employ Cartesian coordinates $(X, Y, Z)$ such that $X=r-r_{0}$ is in the radial direction, $Y=r_{0}\left(\phi-\phi_{0}\right)$ is in the azimuthal direction and $Z$ is in the vertical direction.

For ease of comparison with classical results in the fluid literature, we assume that the flow is incompressible, that it extends from $X=-L$ to $+L$, and that there are rigid walls at the two ends with no-slip boundary conditions. The flow is unbounded along $Y$ and $Z$. 
In the limit $L \ll r_{0}$, the unperturbed velocity corresponds to a linear shear of the form

$$
\vec{V}=\left(0,-\frac{U_{0} X}{L}, 0\right),
$$

where $U_{0}$ is the speed at the two walls. Because of rotation, a Coriolis acceleration acts on the fluid and is described by a frequency

$$
\vec{\omega}=\left(0,0, \Omega_{0}\right), \quad \Omega_{0}=\frac{U_{0}}{q L} .
$$

Here the parameter $q$ is positive (corresponding to angular velocity decreasing with increasing radius in a disk) and describes the radial dependence of $\Omega(r)$ in the accretion disk,

$$
\Omega(r)=\Omega_{0}\left(\frac{r_{0}}{r}\right)^{q} .
$$

Thus, $q=3 / 2$ corresponds to a Keplerian disk and $q=2$ corresponds to a disk with a constant specific angular momentum. For completeness, we note that $q=1$ corresponds to a system with a flat rotation curve and $q=0$ to solid body rotation.

The classical plane Couette flow that is widely discussed in the fluid literature has a finite shear but no Coriolis force. In our model, this corresponds to a finite $U_{0}$ but zero $\Omega_{0}$, i.e., it represents the limit $q \rightarrow \infty$. The accretion disk problem, which is of primary interest in astrophysics, corresponds to finite $q$ in the range $3 / 2$ to 2 . In comparing the present work to the fluid literature, the reader is warned that our radial coordinate $X$ maps to $Y$ in the fluid work, while our $Y$ is their $X$. The notation we use is standard in the astrophysics literature. Below we describe the self-contained set of generalized equations from beginning, for the convenience of the general reader.

\subsection{Perturbations}

The dynamics of a viscous incompressible fluid are described by the Navier-Stokes equation (e.g., Landau \& Lifshitz 1989),

$$
\frac{\partial \vec{V}}{\partial t^{\prime}}+\vec{V} \cdot \nabla^{\prime} \vec{V}+\vec{\omega} \times \vec{\omega} \times \vec{D}+2 \vec{\omega} \times \vec{V}+\nabla^{\prime}\left(\frac{P}{\rho}\right)=\nu \nabla^{\prime 2} \vec{V},
$$

supplemented with the condition of incompressibility,

$$
\nabla^{\prime} \cdot \vec{V}=0
$$


where $t^{\prime}$ is time, $\vec{V}$ is the velocity, $\vec{\omega}$ is the Coriolis vector defined in equation (2), $\nu$ is the kinematic coefficient of viscosity, $\vec{D} \equiv(X, Y, Z), \nabla^{\prime} \equiv(\partial / \partial X, \partial / \partial Y, \partial / \partial Z)$, and $P$ is the pressure. Due to the incompressibility assumption, the density $\rho$ is a constant.

It is convenient to analyse the perturbations in terms of dimensionless variables, $x, y, z, t$, defined by

$$
X=x L, Y=y L, Z=z L, \vec{V}=\vec{U} U_{0}, t^{\prime}=t L / U_{0}
$$

where $\vec{U}$ is a dimensionless velocity

$$
\vec{U}=\left(0, U_{y}, 0\right), \quad U_{y}=U(x)=-x .
$$

Then, by substituting (6) into (4), we obtain

$$
\frac{\partial \vec{U}}{\partial t}+\vec{U} \cdot \nabla \vec{U}+\frac{\hat{k} \times \hat{k} \times \vec{d}}{q^{2}}+\frac{2 \hat{k} \times \vec{U}}{q}+\nabla \bar{p}=\frac{1}{R} \nabla^{2} \vec{U}
$$

where $\bar{p} U_{0}^{2}=P / \rho, \vec{d} \equiv(x, y, z), \nabla \equiv(\partial / \partial x, \partial / \partial y, \partial / \partial z)$, and the Reynolds number $R$ is defined by

$$
R=\frac{U_{0} L}{\nu}
$$

We consider small perturbations in the velocity components of the form: $U_{x} \rightarrow u(x, y, z, t), U_{y} \rightarrow U(x)+v(x, y, z, t), U_{z} \rightarrow w(x, y, z, t)$, and perturbations in the pressure $\bar{p} \rightarrow \bar{p}+p(x, y, z, t)$. The linearized Navier-Stokes and continuity equation for the incompressible fluid then give

$$
\begin{gathered}
\left(\frac{\partial}{\partial t}+U \frac{\partial}{\partial y}\right) u-\frac{2 v}{q}+\frac{\partial p}{\partial x}=\frac{1}{R} \nabla^{2} u \\
\left(\frac{\partial}{\partial t}+U \frac{\partial}{\partial y}\right) v+u \frac{\partial U}{\partial x}+\frac{2 u}{q}+\frac{\partial p}{\partial y}=\frac{1}{R} \nabla^{2} v \\
\left(\frac{\partial}{\partial t}+U \frac{\partial}{\partial y}\right) w+\frac{\partial p}{\partial z}=\frac{1}{R} \nabla^{2} w \\
\frac{\partial u}{\partial x}+\frac{\partial v}{\partial y}+\frac{\partial w}{\partial z}=0
\end{gathered}
$$

Let us rewrite the equations in terms of the $x$ component of the vorticity,

$$
\zeta=\frac{\partial w}{\partial y}-\frac{\partial v}{\partial z}
$$


Combining equations (10)-(13) and simplifying, we obtain

$$
\nabla^{2} p=-2 \frac{\partial U}{\partial x} \frac{\partial u}{\partial y}+\frac{2}{q}\left(\frac{\partial v}{\partial x}-\frac{\partial u}{\partial y}\right)
$$

Eliminating $p$ and $v$ from (10) by use of (13)-(15) we find

$$
\left(\frac{\partial}{\partial t}+U \frac{\partial}{\partial y}\right) \nabla^{2} u-\frac{\partial^{2} U}{\partial x^{2}} \frac{\partial u}{\partial y}+\frac{2}{q} \frac{\partial \zeta}{\partial z}=\frac{1}{R} \nabla^{4} u .
$$

Finally, combining (11) and (12) by use of (14) we obtain

$$
\left(\frac{\partial}{\partial t}+U \frac{\partial}{\partial y}\right) \zeta-\frac{\partial U}{\partial x} \frac{\partial u}{\partial z}-\frac{2}{q} \frac{\partial u}{\partial z}=\frac{1}{R} \nabla^{2} \zeta
$$

where we recall from equation (7) that $U=-x$.

Equations (16) and (17) are the standard Orr-Sommerfeld and Squire equations, respectively, except that they now have additional terms proportional to $2 / q$ because of the inclusion of Coriolis acceleration. We are interested in solving these linear equations with no-slip boundary conditions, i.e., $u=v=w=0$ at the two walls. Equivalently

$$
u=\frac{\partial u}{\partial x}=\zeta=0, \text { at } x= \pm 1 .
$$

Because of translation-invariance of the unperturbed flow in $y$ and $z$, we can decompose the perturbations in terms of Fourier modes in these directions. Also, for convenience, we study the perturbations in terms of $(u, \zeta)$ rather than $(u, v, w)$. Therefore, we write the perturbations as

$$
\begin{aligned}
u(x, y, z, t) & =\hat{u}(x, t) \exp \left[i \vec{k} \cdot \vec{r}_{p}\right], \\
\zeta(x, y, z, t) & =\hat{\zeta}(x, t) \exp \left[i \vec{k} \cdot \vec{r}_{p}\right]
\end{aligned}
$$

where $\vec{r}_{p}[\equiv(y, z)]$ is any radius vector in the $y-z$ plane and $\vec{k} \equiv\left(k_{y}, k_{z}\right)$. By substituting (19) into (16) and (17), we obtain

$$
\begin{aligned}
& \frac{\partial \hat{u}}{\partial t}=-i\left[\mathcal{L}_{o s} \hat{u}+\tilde{\mathcal{L}}_{c o r} \hat{\zeta}\right] \\
& \frac{\partial \hat{\zeta}}{\partial t}=-i\left[\left(\mathcal{L}_{c}+\mathcal{L}_{c o r}\right) \hat{u}+\mathcal{L}_{s q} \hat{\zeta}\right]
\end{aligned}
$$

where

$$
\mathcal{L}_{\text {os }}=-\left(D^{2}-k^{2}\right)^{-1}\left[\left(D^{2}-k^{2}\right)^{2} /(i R)-k_{y} U\left(D^{2}-k^{2}\right)+k_{y} D^{2} U\right]
$$




$$
\begin{aligned}
\mathcal{L}_{c} & =-k_{z} D U, \\
\mathcal{L}_{s q} & =k_{y} U-\left(D^{2}-k^{2}\right) /(i R), \\
\mathcal{L}_{c o r} & =-\frac{2 k_{z}}{q} \\
\tilde{\mathcal{L}}_{c o r} & =-\mathcal{L}_{c o r}\left(D^{2}-k^{2}\right)^{-1}, \\
D & =\partial / \partial x .
\end{aligned}
$$

If we further define

$$
Q=\left(\begin{array}{c}
\hat{u} \\
\hat{\zeta}
\end{array}\right), \quad \mathcal{L}=\left(\begin{array}{cc}
\mathcal{L}_{o s} & \tilde{\mathcal{L}}_{\text {cor }} \\
\mathcal{L}_{c}+\mathcal{L}_{\text {cor }} & \mathcal{L}_{\text {sq }}
\end{array}\right),
$$

equation (20) reduces to the form

$$
\frac{\partial Q}{\partial t}=-i \mathcal{L} Q
$$

which we need to solve to obtain the eigenvectors and corresponding eigenvalues. Since the set of eigenmodes for this bounded flow problem is discrete and complete, we can write the solution to (23) in terms of an eigenfunction expansion,

$$
\begin{aligned}
Q(x, t) & =\sum_{j=1}^{\infty}\left[A_{j} \exp \left(-i \lambda_{j} t\right) \tilde{Q}_{j}^{1}(x)+B_{j} \exp \left(-i \mu_{j} t\right) \tilde{Q}_{j}^{2}(x)\right] \\
\tilde{Q}_{j}^{1}(x) & =\left(\begin{array}{c}
\tilde{u}_{j}^{1}(x) \\
\tilde{\zeta}_{j}^{1}(x)
\end{array}\right), \\
\tilde{Q}_{j}^{2}(x) & =\left(\begin{array}{c}
\tilde{u}_{j}^{2}(x) \\
\tilde{\zeta}_{j}^{2}(x)
\end{array}\right),
\end{aligned}
$$

where $\left(\lambda_{j}, \tilde{Q}_{j}^{1}(x)\right)$ is the Orr-Sommerfeld eigensystem ${ }^{4}$ and $\left(\mu_{j}, \tilde{Q}_{j}^{2}(x)\right)$ is the Squire eigensystem. Formally merging the two systems, we can rewrite (24) as

$$
\begin{aligned}
Q(x, t) & =\sum_{j=1}^{\infty} C_{j} \exp \left(-i \sigma_{j} t\right) \tilde{Q}_{j}(x), \\
\tilde{Q}_{j}(x) & =\left(\begin{array}{c}
\tilde{u}_{j}(x) \\
\tilde{\zeta}_{j}(x)
\end{array}\right),
\end{aligned}
$$

where half the indices $j$ correspond to the Orr-Sommerfeld modes and the other half of $j$ correspond to the Squire modes, and $\sigma_{j}=\sigma_{R j}+i \sigma_{I j}$. Therefore, for the $j$ th mode, (23) reduces to

$$
\mathcal{L} \tilde{Q}_{j}=\sigma_{j} \tilde{Q}_{j}
$$

\footnotetext{
${ }^{4} \mathrm{~A}$ complete set of eigenvalues and eigenvectors is called the eigensystem.
} 
To calculate the set of eigenvalues and eigenvectors, we convert the differential operator $\mathcal{L}$ into an $N \times N$ matrix in a finite-difference representation and we then compute the eigenvalues and eigenvectors of the matrix. The required order $N$ of the matrix for adequate accuracy depends on the physical parameters of the problem (mainly $R$ and also $k_{y}, k_{z}$ ). For the calculations presented here, we used $N$ in the range $200-300$ (i.e., each of the blocks $\mathcal{L}_{o s}, \mathcal{L}_{s q}, \mathcal{L}_{c}$ and $\mathcal{L}_{\text {cor }}$ had a size in the range 100 to 150 ).

\subsection{Energy Growth}

The eigenvalues $\sigma_{j}$ and the corresponding eigenvectors $\tilde{Q}_{j}$ for plane Couette flow have been studied by a number authors (e.g. Orszag 1971; Romanov 1973; Farrell 1988; Reddy \& Henningson 1993) who have shown that there are no exponential growing eigenmodes in the system. That is, for no choice of the parameters is there an eigenvalue with positive $\sigma_{I}$. Figure 1a shows such a typical eigenspectrum: the case shown has $R=2000$ and $k_{y}=k_{z}=1$. For comparison Fig. 1b shows the eigenspectrum of a disk with constant angular momentum $q=2$, and Fig. 1c a Keplerian disk $q=3 / 2$. To the best of our knowledge, this one to one comparison of eigenspectra between standard plane Couette flow, a constant angular momentum flow and a Keplerian flow has not been reported earlier. It is clear that none of these flows has any growing eigenmode and so all three systems are linearly stable. It should also be noticed that the eigenspectrum for plane Couette flow is very similar to that of a constant angular momentum flow. We explore this similarity further in $\S \S 3,4$.

One of the most important features of plane Couette flow (and its governing linear operator) is that the eigenmodes of the system are close to linearly dependent in nature i.e. they are non-normal in nature. Because of this, even in the absence of any exponentially growing mode in the system, it is possible to have a large transient growth in the energy of certain perturbations (Butler \& Farrell 1992; Trefethen et al. 1993). This growth occurs in the absence of non-linear effects and is believed to play an important role in the transition from laminar to turbulent flow.

Following previous authors (e.g. Trefethen et al. 1993; Schmid \& Henningson 1994) we define the perturbed energy density as

$$
E=\frac{1}{2 V} \int_{-1}^{1} \int_{0}^{a} \int_{0}^{b}\left(u^{2}+v^{2}+w^{2}\right) d z d y d x,
$$

where $a=2 \pi / k_{y}, b=2 \pi / k_{z}$, and $V=2 a b$ is the integration volume. We then seek to maximize the growth in this quantity. Recalling the formal solution of (23) in matrix form,

$$
Q(x, t)=\exp [-i \mathcal{L} t] Q(x, 0),
$$



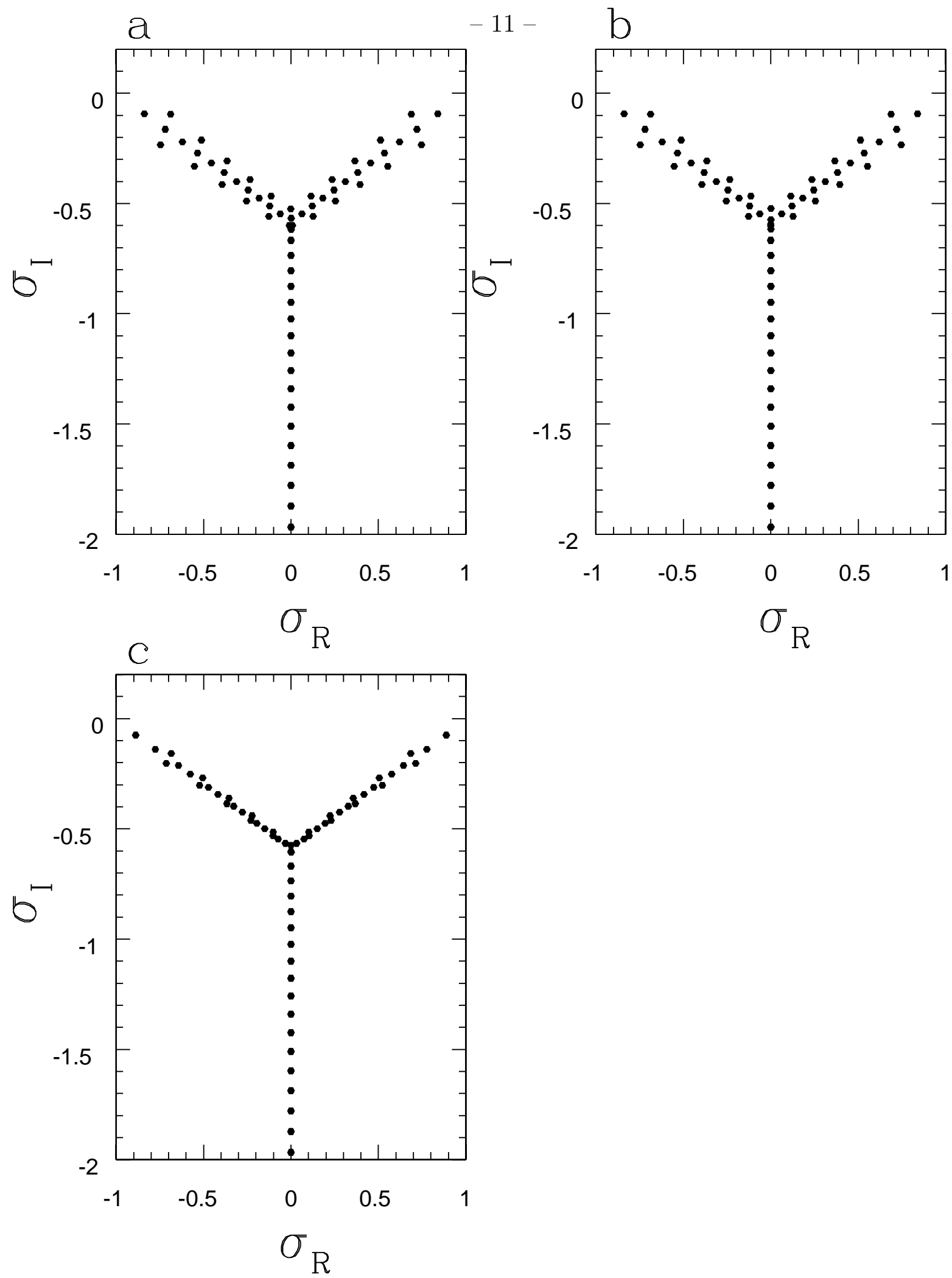

Fig. 1. - Eigenspectra of (a) plane Couette flow, (b) constant angular momentum disk $(q=2)$, and (c) Keplerian disk $(q=3 / 2)$, for $k_{y}=k_{z}=1, R=2000$. 
the maximum growth in the perturbed energy can be expressed as

$$
G\left(k_{y}, k_{z}, R, t\right)=\operatorname{maximum}\left(\frac{\|Q(\cdot, t)\|_{2}^{2}}{\|Q(\cdot, 0)\|_{2}^{2}}\right)=\|\exp [-i \mathcal{L} t]\|_{2}^{2},
$$

where $\|\ldots\|_{2}$ signifies the norm of the respective quantity and the subscript 2 specifies the 2-norm or Euclidian norm. The 2-norm of the matrix can be evaluated by means of a singular value decomposition. Then, for a given $t$, the square of the highest singular value is the maximum energy growth, $G_{\max }(t)$, for that time. Physically, by "maximum" we mean that we consider all possible initial perturbations $Q(x, 0)$ and choose that function that maximizes the growth of energy at time $t$. The corresponding energy growth factor is $G\left(k_{y}, k_{z}, R, t\right)$. When $t=0$, by definition $G\left(k_{y}, k_{z}, R, t\right)=1$, implying no growth. For given $t$, we maximize $G\left(k_{y}, k_{z}, R, t\right)$ by writing $Q(x, 0)$ as a linear combination of the eigenmodes of the system as in equation (25) and optimizing the coefficients $C_{j}$. The details are given in the Appendix.

We should mention that to evaluate the growth one does not need to include all the eigenmodes in the computation. It has been shown by Reddy \& Henningson (1993) that only a limited number, $K / 2$, of the Orr-Sommerfeld and Squire modes, viz., those with the largest (i.e., least negative) $\sigma_{I}$ values, are responsible for the growth. The remaining modes decay too rapidly to provide much growth. Therefore (25) can be rewritten as

$$
Q_{K}(x, t)=\sum_{j=1}^{K} C_{j} \exp \left(-i \sigma_{j} t\right) \tilde{Q}_{j}(x)=\tilde{Q} \exp \left[-i \Sigma_{K} t\right] C
$$

and the corresponding growth is $G_{K}\left(k_{y}, k_{z}, R, t\right)$. Here $\tilde{Q}$ and $C$ are $N \times K$ and $K \times 1$ matrices respectively and $\Sigma_{K}$ is a $K \times K$ diagonal matrix consisting of the top $K$ eigenvalues (top $K / 2$ of Orr-Sommerfeld and $K / 2$ of Squire eigenvalues). For the calculations presented in this paper, we generally used $K \leq 60$.

The growth $G\left(k_{y}, k_{z}, R, t\right)$ defined above is a function of four parameters. In various places in the paper we consider different kinds of maxima of this function. For instance, for fixed $k_{y}, k_{z}, R$, we could maximize $G$ with respect to time $t$, and thereby determine the maximum growth $G_{\max }\left(k_{y}, k_{z}, R\right)$. This is the quantity that is plotted as contours in Figures 2 and 4 . Or, we may wish to hold one of the components of the wavevector fixed, e.g., $k_{y}=0$ (see Table $1, \S 4.1$, also Figure 6 for other values of $k_{y}$ ) or $k_{z}=0(\S 4.2)$, and optimize the growth with respect to the other component of the wavevector and the time; this gives maximum growth factors such as $G_{\max }\left(k_{y}=0, R\right)$ and $G_{\max }\left(k_{z}=0, R\right)$. Finally, for a given $R$, we could optimize over all the other parameters to calculate $G_{\max }(R)$. This is the quantity of most interest, and is shown for instance in Tables 1, 2, and Figures 3, 5. 


\section{Numerical Results}

\subsection{Plane Couette Flow and Constant Specific Angular Momentum Flow}

In the previous section, we showed that the eigenspectra of plane Couette flow and a constant angular momentum disk $(q=2)$ are very similar. Here we show that the maximum growths are also similar. Figures $2 \mathrm{a}$,b and $2 \mathrm{c}, \mathrm{d}$ show contours of constant $G_{\max }$ in the $\left\{k_{y}, k_{z}\right\}$ plane for plane Couette flow and a $q=2$ disk respectively for two values of $R$ (500,2000). The maximum growth values for these two cases and for other values of $R$ are found in Fig. 3. We see that the values are very similar for the two flows. Whereas for $q=2$ the maximum growth occurs exactly on the $k_{z}$ axis $\left(k_{y}=0\right)$, for plane Couette flow it is slightly off the axis, though by a progressively smaller amount with increasing $R$. This deviation in location of the occurrence of maximum growth in the $k_{y}-k_{z}$ plane for a constant angular momentum disk compared to plane Couette flow was completely unnoticed earlier, to the best of our knowledge. In cases of a large $R$, the best growth values for $k_{y}=0$ compared to $k_{y} \sim 0$ (but $\neq 0$ ) do not have any practical difference. But for a small $R$ the difference is important as in this case the maximum growth factor itself is small (see Fig. 3). Therefore, in presence of finite molecular viscosity this result has a physical implication to fluid dynamics, though in the accretion disk when $R$ is always expected to be very large this may not be an important issue.

In Figure 3, we show the variation of the maximum growth $G_{\max }$ and the corresponding time $t_{\max }$ at which the maximum growth occurs as functions of $R$ for plane Couette flow and $q=2$. We see that $G_{\max }$ varies as $R^{2}$ and $t_{\max }$ as $R$ in both cases, with very similar values, again indicating the similarity of the two flows. However for plane Couette flow, growth maximizes for $k_{z} \sim 1.6$, while for a $q=2$ disk it happens at $k_{z}=1.66$. Moreover, in plane Couette Flow, the optimum $k_{y}$ scales as $1 / R$, already noticed by earlier authors (e.g. Butler \& Farrell 1992), whereas for a $q=2$ flow the optimum $k_{y}=0$.

In the case of a constant angular momentum disk the epicyclic frequency of the disk becomes zero which makes the basic structure of the system very similar to that of plane Couette flow (compare the equation set (10)-(12) as well as (16) and (17) for plane Couette flow and constant angular momentum flow). This was already noticed by Balbus, Hawley \& Stone (1996) and Hawley, Balbus \& Winters (1999), who found from numerical simulations that these two flows are equally susceptible to hydrodynamic turbulence. We explore the physics of this similarity further in $\S 4$. 

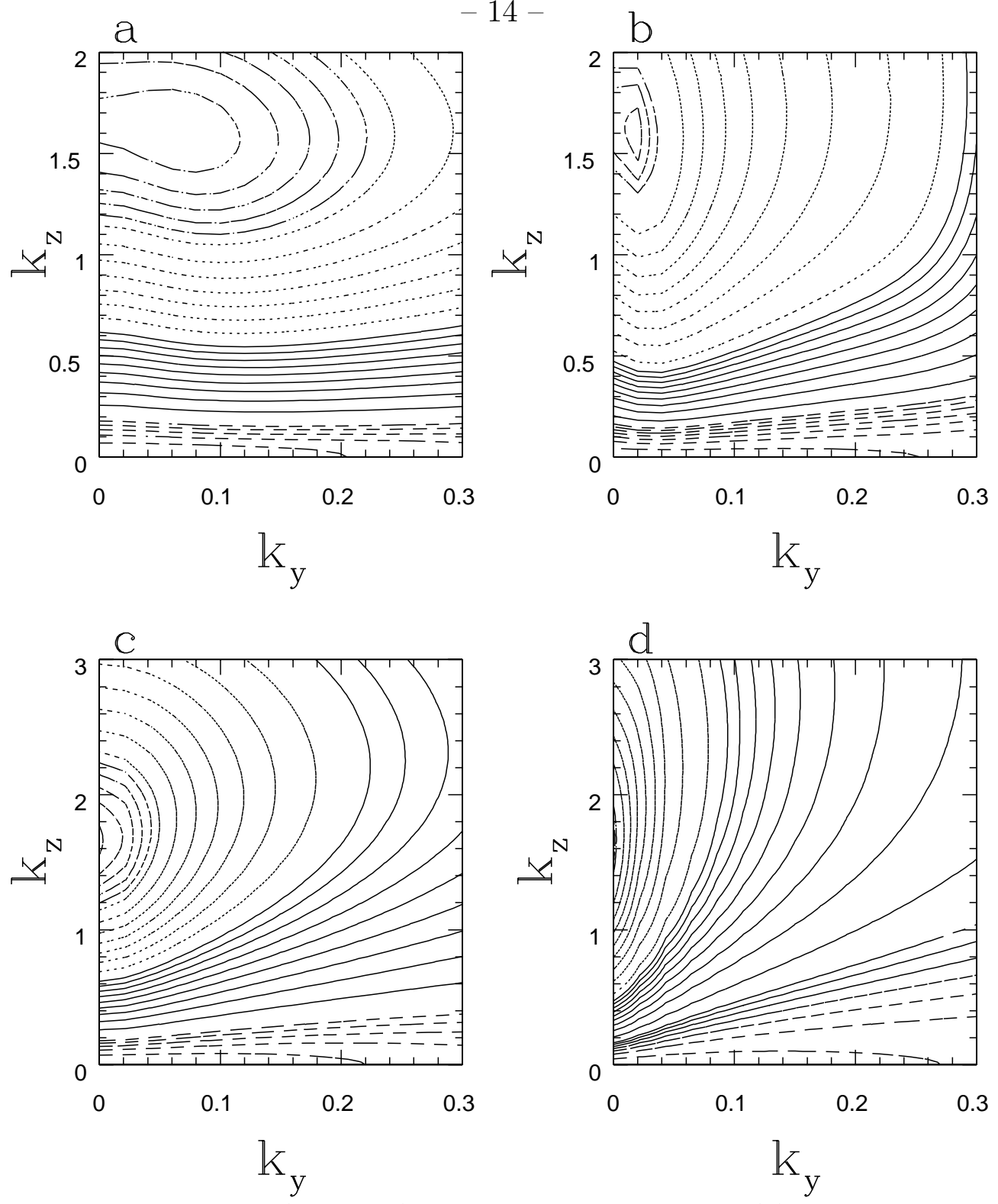

Fig. 2.- Contours of $G_{\max }\left(k_{y}, k_{z}, R\right)$ in the $k_{y}-k_{z}$ plane. (a) Plane Couette flow for $R=500$ : dashed contours correspond to $G_{\max }=2,4, \ldots, 10$, solid contours to $G_{\max }=$ $20,30, \ldots, 100$, dotted contours to $G_{\max }=120,140, \ldots, 240$, and dot-dashed contours to $G_{\max }=250,260, \ldots, 290$. (b) Plane Couette flow for $R=2000$ : dashed contours correspond to $G_{\max }=10,30, \ldots, 130$, solid contours to $G_{\max }=200,300, \ldots, 1000$, dotted contours to $G_{\max }=1200,1600, \ldots, 4000$, and dot-dashed contours to $G_{\max }=4500,4600,4700 . \quad$ (c) Constant angular momentum disk $(q=2)$ for $R=500$ : dashed contours correspond to $G_{\max }=2,4, \ldots, 10$, solid contours to $G_{\max }=20,30, \ldots, 100$, dotted contours to $G_{\max }=$ $120,140, \ldots, 240$, and dot-dashed contours to $G_{\max }=250,260, \ldots, 290$. (d) Constant angular momentum disk for $R=2000$ : dashed contours correspond to $G_{\max }=10,30, \ldots, 130$, solid contours to $G_{\max }=200,300, \ldots, 1000$, dotted contours to $G_{\max }=1200,1600, \ldots, 4000$, and dot-dashed contours to $G_{\max }=4500,4600$. 


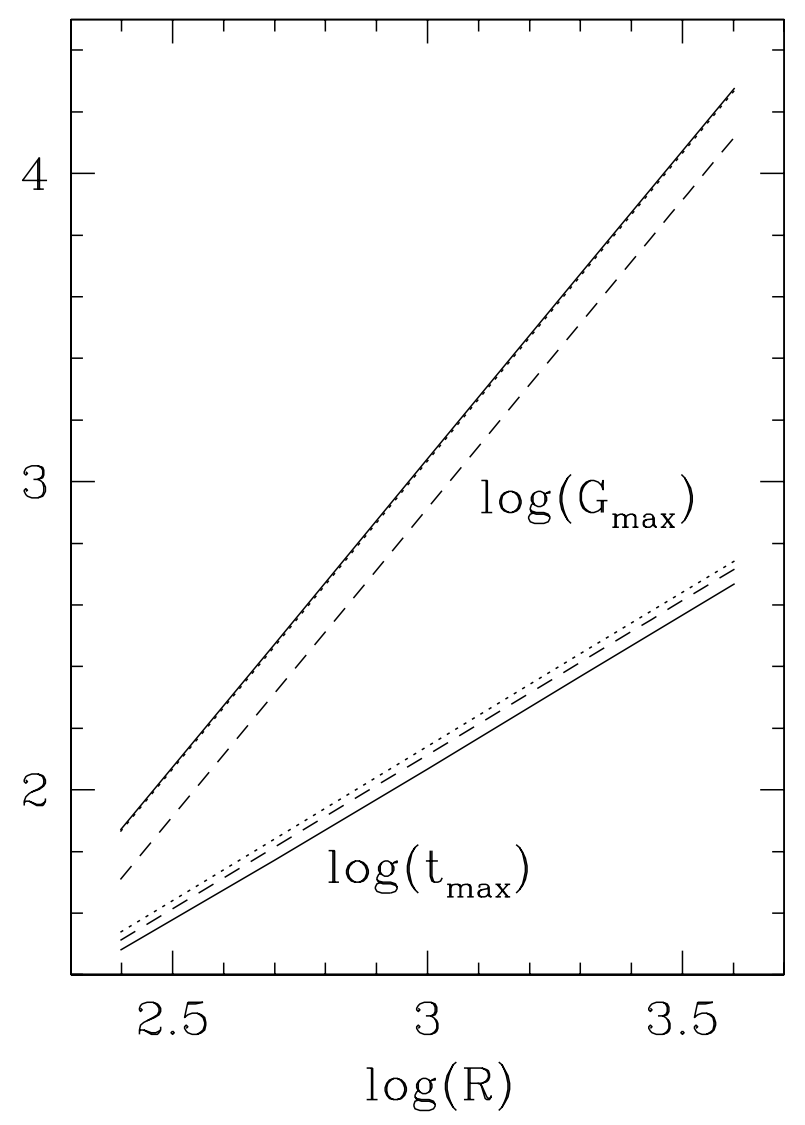

Fig. 3.- $G_{\max }(R)$ and $t_{\max }(R)$ as functions of Reynolds number $R$. Solid lines correspond to plane Couette flow and dotted lines to a constant angular momentum disk $(q=2)$. The dashed lines show the analytic result discussed in $\S 4.1 .1$. 


\subsection{Effect of Non-Zero Epicyclic Frequency}

The epicyclic frequency is given by

$$
\kappa=\sqrt{2(2-q)} \Omega
$$

which is zero for $q=2$ and is non-zero (and real) for any $q<2$. Figures $2(\mathrm{c})$,(d) show that, when $q=2$, the maximum growth occurs for axisymmetric perturbations with vertical structure (hereafter we refer as "vertical perturbation"), i.e. $k_{y}=0, k_{z} \neq 0$. We begin this section by exploring what happens to such vertical perturbations when $q<2$. Note that most of the fluid literature is devoted to plane Couette flow with no Coriolis force. In the astrophysics literature, Yecko (2004) mainly concentrated on the Keplerian disk $(q=1.5)$ and plane Couette flow, whereas we consider the full range, $1.5 \leq q \leq 2$.

Table 1 shows the maximum growth for vertical perturbations for four values of $q$ : $1.99,1.9,1.7,1.5$. We see that, as $q$ decreases, the growth falls dramatically, and so does the time at which the maximum occurs. For a Keplerian flow, the growth factor is under 4. Moreover, numerical experiments show that the growth is insensitive to the value of $R$. In other words, the growth is not limited by viscosity but rather by the dynamics itself. We explore the reasons for this in $§ 4$.1. Similar results are discussed in AMN05, using a Lagrangian picture.

\section{Table 1}

Energy Growth Factors of Disks for $R=2000$ and $k_{y}=0$

\begin{tabular}{cccc}
\hline \hline$q$ & $k_{z}$ & $G_{\max }\left(k_{y}=0, R=2000\right)$ & $t_{\max }\left(k_{y}=0, R=2000\right)$ \\
\hline \hline 1.5 & 2.5 & 3.84 & 2.8 \\
\hline 1.7 & 2.4 & 6.31 & 4.1 \\
\hline 1.9 & 2.1 & 18.16 & 8.3 \\
\hline 1.99 & 1.9 & 153.4 & 27.8 \\
\hline 2 & 1.66 & 4661 & 277 \\
\hline \hline
\end{tabular}

We next remove the restriction to vertical perturbations and consider general $k_{y}, k_{z}$. Figure 4a shows contours of constant growth for $q=1.99$ for $R=2000$. Even though the value of $q$ is only very slightly different form that used in Figure $2 \mathrm{~d}(q=2)$, nevertheless we see a dramatic change. The main qualitative difference between the two cases is that the epicyclic frequency is zero for $q=2$ but is (slightly) non-zero for $q=1.99$. Still, this small changes causes a major modification of the results, showing what a dominant effect the epicyclic frequency has on the fluid dynamics. The other panels in Fig. 4 show results for other values of $q$. It is interesting to see how the location of the maximum in 
the $k_{y}-k_{z}$ plane changes as the system approaches the Keplerian regime, and also how the magnitude of the growth reduces. This change in location of maximum growth as a function of $q$ in the $k_{y}-k_{z}$ plane has not been noted earlier, to the best of our knowledge. For $q=2$ the maximum energy growth is a factor of 4600 and occurs on the $k_{y}=0$ line (Fig. 2d) while for $q=1.5$ the maximum growth is only 22 and occurs on the $k_{z}=0$ line (Fig. 4d). Therefore for a constant angular momentum disk, we need to include vertical structure in the perturbations to maximize the energy growth, whereas for a Keplerian disk a 2-dimensional analysis is sufficient. Table 2 lists the maximum energy growth factors for $q=1.5-2$ when $R=2000$.

Figures $5 \mathrm{a}$ and $5 \mathrm{~b}$ show respectively the variation of the maximum growth and the time at which the maximum growth occurs as a function of Reynolds number in a Keplerian disk. It is seen that for large $R, G_{\max }$ scales as $R^{2 / 3}$ and $t_{\max }$ as $R^{1 / 3}$. This suggests that, even though the growth is modest for the values of $R$ we have considered, if we go to sufficiently large values of $R$, very large energy growth might still be possible. This is of interest because the Reynolds number of a cold accretion disk is very high (many orders of magnitude higher than the values considered in this work), so turbulence could be generated in such systems. Due to numerical constraints our current results are limited to $R \leq 10^{4}$; however, this range captures most of the basic features of the growth. Yecko (2004) used a superior spectral code and was able to go to much larger values of $R$.

Figure 6 shows how $G_{\max }$ and $t_{\max }$ scale with $k_{y}$ at a given $R$. The maximum growth is achieved at $k_{y} \sim 1.2$. At smaller $k_{y}, G_{\max }$ scales as $k_{y}^{2 / 3}$, while at larger $k_{y}, G_{\max }$ decreases as $\sim 1 / k_{y}$ or $k_{y}^{-4 / 3}$. Also $t_{\max }$ scales as $k_{y}^{-2 / 3}$ at large $t$. Yecko (2004) and Umurhan \& Regev (2004) identified the scaling of $G_{\max }$ with $R$ for a Keplerian disk, but the other scaling relations have not been discussed before. We also derive all the scaling relations analytically, for the first time, in $\S 4$. A detailed understanding of these scalings is given in $\S 4.2$. Identical scaling relations are also derived by AMN05. 
Table 2

Maximum Energy Growth for Disks with Various Values of $q$ and $R=2000$

\begin{tabular}{ccccc}
\hline \hline$q$ & $k_{y}$ & $k_{z}$ & $G_{\max }(R=2000)$ & $t_{\max }(R=2000)$ \\
\hline \hline 1.5 & 1.2 & 0 & 21.67 & 11 \\
\hline 1.7 & 1.05 & 0.6 & 23 & 12.4 \\
\hline 1.9 & 0.34 & 0.96 & 32.33 & 19.8 \\
\hline 1.99 & 0.14 & 1.64 & 174.43 & 34.3 \\
\hline 2 & 0 & 1.66 & 4661 & 277 \\
\hline \hline
\end{tabular}

\subsection{Nature of the Growing Perturbations in a Keplerian Flow}

We have seen that for a Keplerian flow the maximum growth occurs for $k_{y} \sim 1.2$, $k_{z}=0$. Figures 7 and 8 show the development with time of the perturbed velocity component $u(x, y)$ corresponding to $R=500,4000$, respectively, optimized for the best growing mode. In each case, we show snapshots corresponding to four times: $t=0, t_{\max } / 2$, $t_{\max }, 3 t_{\max } / 2$. The perturbations are seen to resemble plane waves that are frozen in the shearing flow. The initial perturbation at $t=0$ is a leading wave with negative $k_{x}$ and with $\left|k_{x}\right| \gg k_{y}$. With time, the wavefronts are straightened out by the shear, until at $t=t_{\max }$, the wavefronts are almost radial and $k_{x} \sim 0$. At yet later times, the wave becomes trailing and the energy also decreases. The perturbations are very similar to the growing perturbation described by Chagelishvili et al. (2003) and Umurhan \& Regev (2004). However, those authors (and also AMN05) considered an infinite system whereas our fluid is confined to a box of size $2 L$ in the $x$ direction. Figure 9 shows the optimum growth of the energy $G(t)$ as a function of time for the two perturbations whose time evolution for the best growing modes are shown in Figures 7 and 8.

\section{Physical Interpretation of the Numerical Results}

In this section we attempt to understand via an analytical approach the numerical results of the previous sections. We also like to derive the scaling relations described in the previous sections analytically. We show that the analytical solutions match the numerical results well. In the interest of clarity, we work with the original dimensioned equations. Thus $X$ (going from $-L$ to $+L$ ), $Y, Z$ are our coordinates, and we write the corresponding components of the wavevector as $k_{X}, k_{Y}, k_{Z}$, respectively. We use $t$ for time (called $t^{\prime}$ in $\S 2.1)$. 

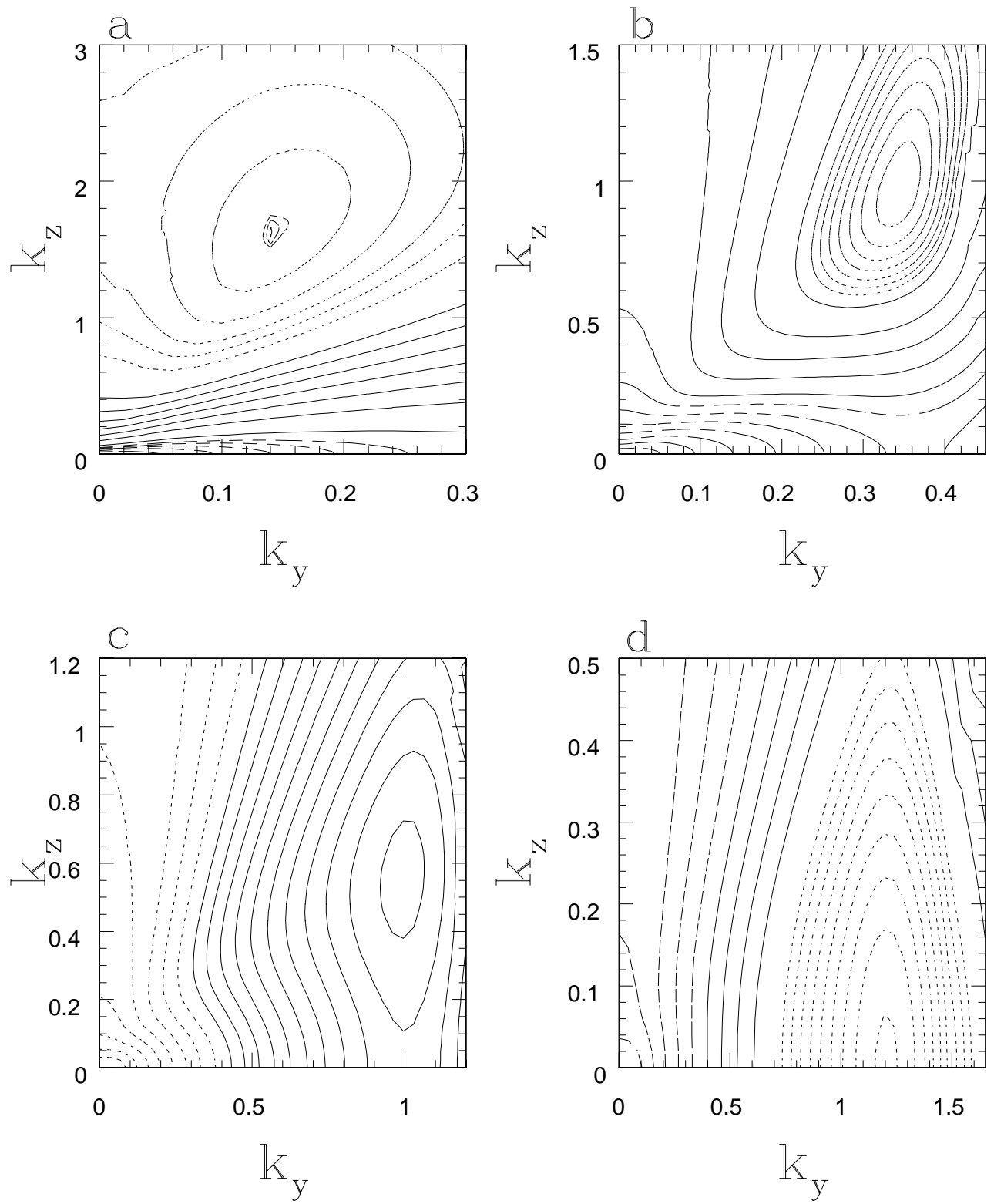

Fig. 4.- Contours of $G_{\max }\left(k_{y}, k_{z}, R\right)$ for disks with different values of $q$ and $R=2000$. (a) $q=1.99$ : dashed contours correspond to $G_{\max }=2,4, \ldots, 10$, solid contours to $G_{\max }=15,30, \ldots, 105$, dotted contours to $G_{\max }=130,140, \ldots, 170$, and dot-dashed contours to $G_{\max }=174,174.2,174.4$. (b) $q=1.9$ : dashed contours correspond to $G_{\max }=2,4, \ldots, 14$, solid contours to $G_{\max }=16,19, \ldots, 28$, and dotted contours to $G_{\max }=29,29.5, \ldots, 32$. (c) $q=1.7$ : dotted contours correspond to $G_{\max }=2,3, \ldots, 9$, and solid contours to $G_{\max }=11,12.2, \ldots, 23$. (d) $q=1.5$ : dashed contours correspond to $G_{\max }=2,3, \ldots, 6$, solid contours to $G_{\max }=7,9, \ldots, 13$, and dotted contours to $G_{\max }=17.5,18, \ldots, 21.5$. 

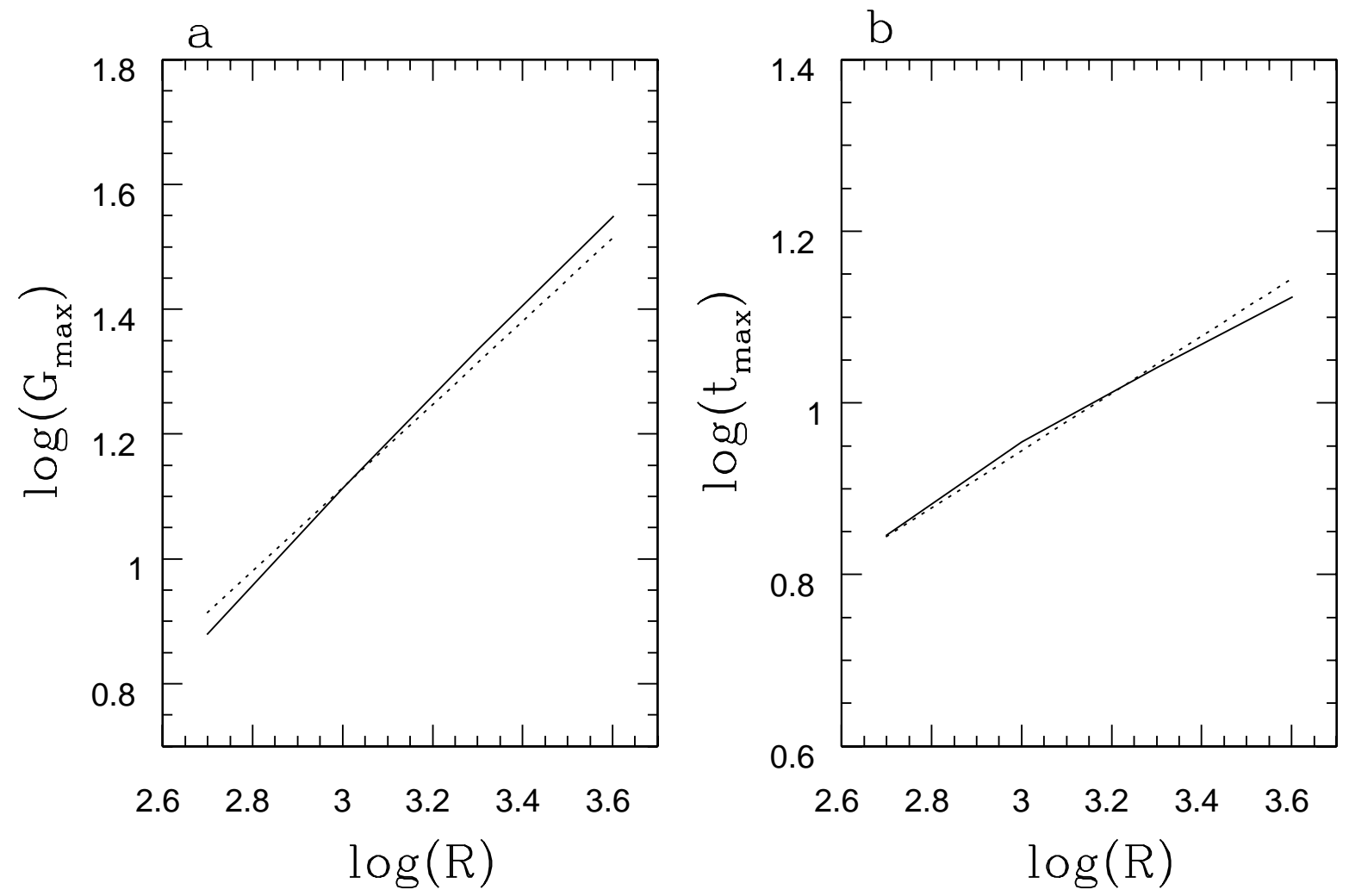

Fig. 5.- Solid lines show (a) $G_{\max }(R)$, and (b) $t_{\max }(R)$, as functions of $R$ for $q=1.5$. Dotted lines correspond to the analytical result for $k_{x, \min }=1.7$ discussed in $\S 4.2$. 


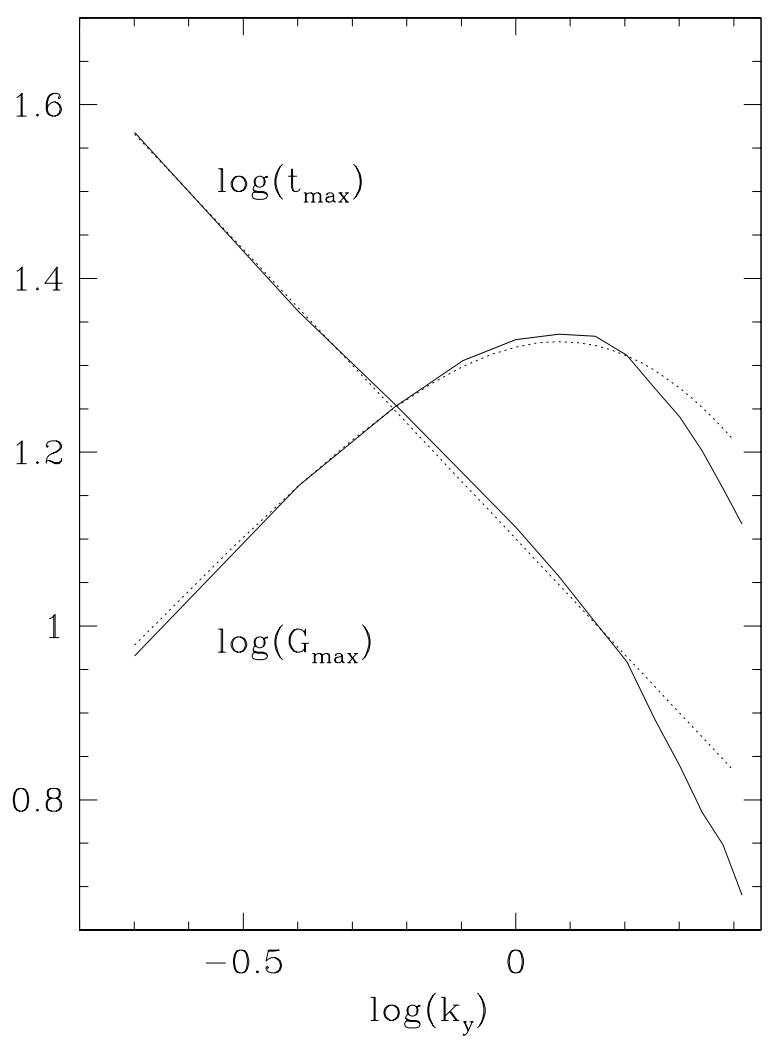

Fig. 6.- $G_{\max }\left(k_{y}, R\right)$ and $t_{\max }\left(k_{y}, R\right)$ as functions of $k_{y}$ for $q=1.5$ and $R=2000$. The dotted lines correspond to the analytical result discussed in $§ 4.2$. 

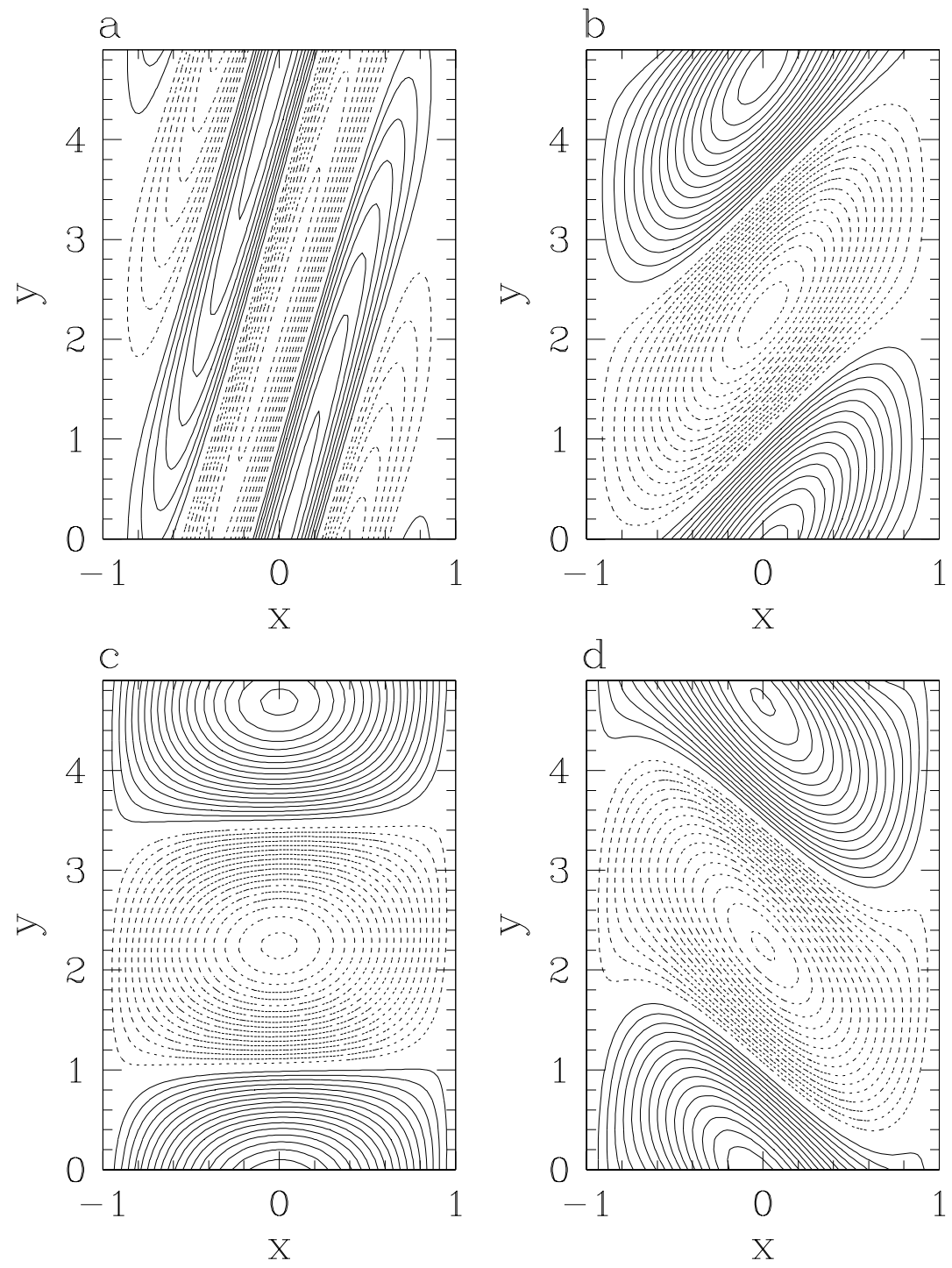

Fig. 7.- Shows the development of the perturbed velocity $u(x, y)$ as a function of time for the best growing energy optimal in a Keplerian flow with $R=500$. The perturbation has $k_{y}=1.29, k_{z}=0$, and the maximum growth is achieved at $t_{\max }(R=500)=6.6$. The four panels correspond to (a) $t=0$, (b) $t=t_{\max } / 2=3.3$, (c) $t=t_{\max }=6.6$, (d) $t=3 t_{\max } / 2=9.9$. Solid and dotted contours correspond to positive and negative values of $u$ respectively. 

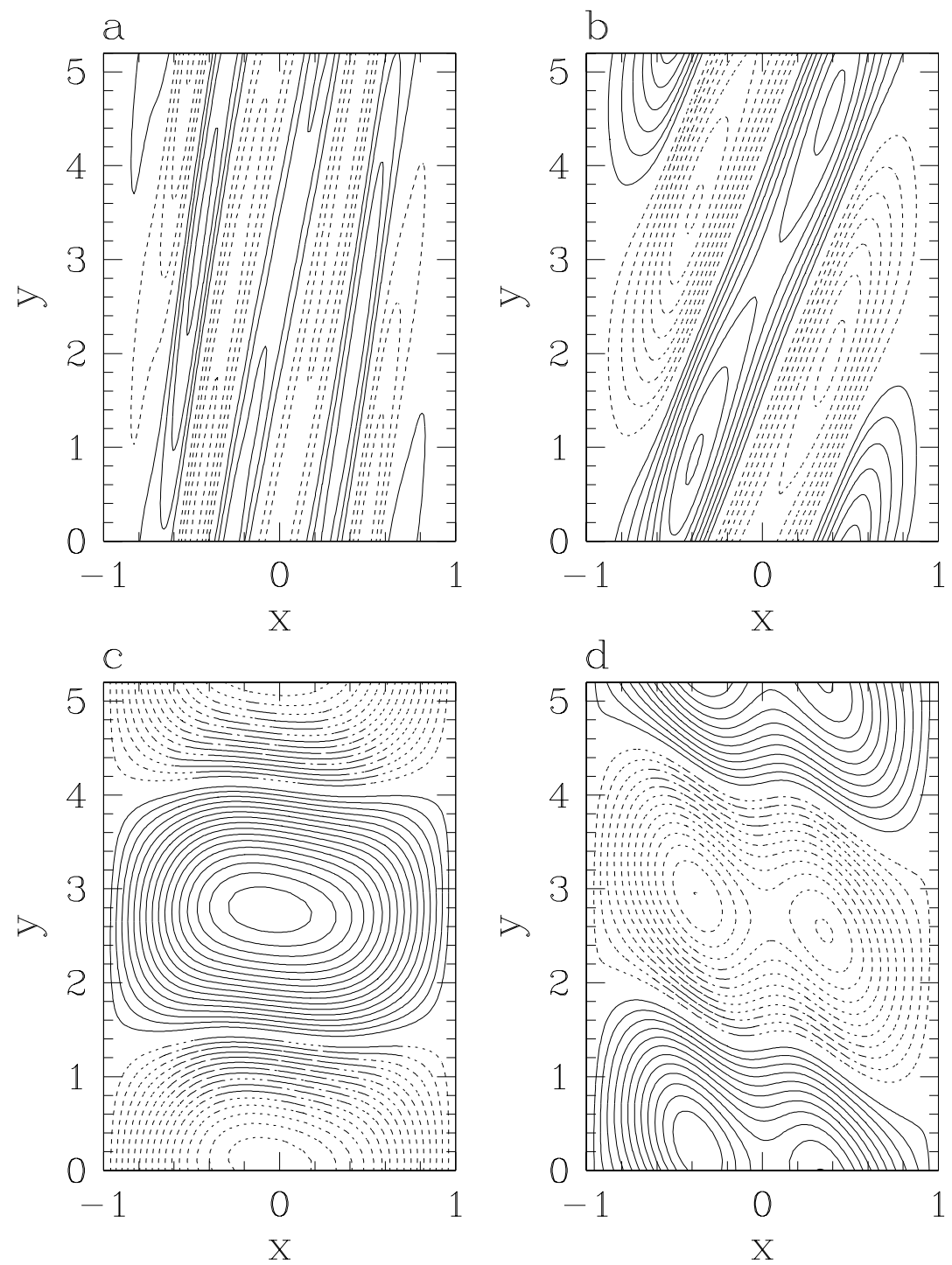

Fig. 8.- Same as Fig. 7 but for $R=4000$. Here $k_{y}=1.2, k_{z}=0, t_{\text {max }}(R=4000)=13.3$, and the four panels correspond to (a) $t=0$, (b) $t=t_{\max } / 2=6.65$, (c) $t=t_{\max }=13.3$, (d) $t=3 t_{\max } / 2=19.95$. Solid and dotted contours correspond to positive and negative values of $u$ respectively. 


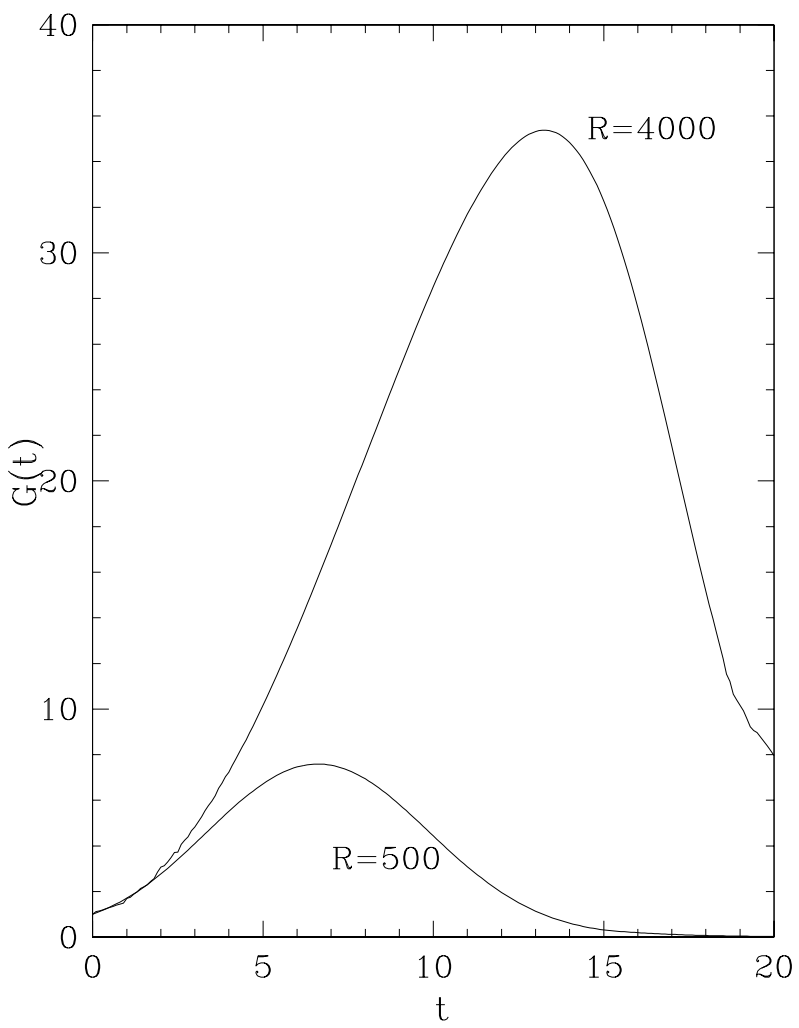

Fig. 9.- Growth of the perturbed energy $G(t)$ as a function of time for the cases shown in Figs. 7 and 8 . 
Let us define the shear frequency $2 A$ and the vorticity frequency $2 B$ as follows (Narayan, Goldreich \& Goodman 1987),

$$
2 A=-q \Omega, \quad 2 B=2(A+\Omega)=(2-q) \Omega .
$$

Then the three components of the momentum equation and the incompressibility condition give

$$
\begin{gathered}
\frac{d u}{d t}=2 \Omega v-\frac{\partial \hat{p}}{\partial X}+\nu \nabla^{\prime 2} u \\
\frac{d v}{d t}=-2 B u-\frac{\partial \hat{p}}{\partial Y}+\nu \nabla^{\prime 2} v \\
\frac{d w}{d t}=-\frac{\partial \hat{p}}{\partial Z}+\nu \nabla^{\prime 2} w \\
\frac{\partial u}{\partial X}+\frac{\partial v}{\partial Y}+\frac{\partial w}{\partial Z}=0
\end{gathered}
$$

where $\hat{p}=P / \rho$. The Lagrangian time derivative $d / d t$ is given by

$$
\frac{d}{d t}=\frac{\partial}{\partial t}-q \Omega X \frac{\partial}{\partial Y}
$$

The numerical results described in $\S 3$ showed that plane Couette flow and $q=2$ flow both have maximum energy growth for perturbations with vertical structure, whereas Keplerian $q=3 / 2$ flow has maximum growth for two-dimensional perturbations with no vertical structure. We analyse these two cases separately.

\subsection{Vertical Perturbations}

To begin with, let us ignore the walls and assume plane wave solutions of the form

$$
u(X, Y, Z, t)=u(t) \exp \left(i k_{X} X+i k_{Y} Y+i k_{Z} Z\right), \text { etc.. }
$$

Furthermore, since perturbations with $k_{Y}=0$ (equivalent to $k_{y}=0$ ) were seen to grow robustly for both plane Couette flow and $q=2$, let us assume $k_{Y}=0$. For such perturbations, $d / d t=\partial / \partial t$.

\subsubsection{Plane Couette Flow}

For plane Couette low, we set $2 \Omega=0$ in equation (33) and $2 B=2 A$ in equation (34). We can show that the fastest growing plane wave perturbation for a given $k_{X}, k_{Z}$ takes the 
form

$$
\begin{gathered}
u=u_{0} \exp \left[i k_{X} X+i k_{Z} Z-\nu\left(k_{X}^{2}+k_{Z}^{2}\right) t\right], \\
v=-2 A t u_{0} \exp \left[i k_{X} X+i k_{Z} Z-\nu\left(k_{X}^{2}+k_{Z}^{2}\right) t\right], \\
w=-\frac{k_{X}}{k_{Z}} u_{0} \exp \left[i k_{X} X+i k_{Z} Z-\nu\left(k_{X}^{2}+k_{Z}^{2}\right) t\right], \\
\hat{p}=0,
\end{gathered}
$$

where $u_{0}$ is an arbitrary amplitude. The ratio of energy at time $t$ to the initial energy is then given by

$$
G(t)=\frac{u^{2}(t)+v^{2}(t)+w^{2}(t)}{u^{2}(0)+v^{2}(0)+w^{2}(0)}=\left[1+\frac{k_{Z}^{2}}{k_{X}^{2}+k_{Z}^{2}}(2 A t)^{2}\right] \exp \left[-2 \nu\left(k_{X}^{2}+k_{Z}^{2}\right) t\right] .
$$

Since we are interested in flows that have perturbations with large growth, let us ignore the 1 in the first factor. Then, the time at which the energy is maximum is given by

$$
t_{\text {max }}=\frac{1}{\nu\left(k_{X}^{2}+k_{Z}^{2}\right)}
$$

and the corresponding energy growth factor is

$$
G_{\max }=\left(\frac{2 A}{\nu}\right)^{2} e^{-2} \frac{k_{Z}^{2}}{\left(k_{X}^{2}+k_{Z}^{2}\right)^{3}} .
$$

The problem we have analysed in $\S 3$ is a flow with walls at $X= \pm L$ with no-slip boundary conditions. In the absence of viscosity, the simplest solution to this problem is

$$
u=-\frac{k_{Z}}{k_{X}} w=u_{0}\left(1+\cos \frac{\pi X}{L}\right) \exp \left(i k_{Z} Z\right), \quad v=\hat{p}=0
$$

which can be seen by inspection to satisfy the boundary conditions at $X= \pm L$. This solution is the sum of a plane wave with $k_{X}=0$ and amplitude $u_{0}$, and two waves with $k_{X}= \pm \pi / L$ and amplitude $u_{0} / 2$. Roughly, we expect that the solutions for $t_{\max }, G_{\max }$ we wrote down earlier for a single plane wave will be approximately correct provided we set $k_{X}^{2}$ equal to $(\pi / L)^{2} / 2$, i.e., the mean of 0 and $(\pi / L)^{2}$. Noting that the Reynolds number is given by

$$
R=\frac{|2 A| L^{2}}{\nu}
$$

we find that the maximum growth factor is given by

$$
G_{\max }\left(k_{Z}, R\right)=\frac{R^{2} k_{Z}^{2} L^{2} e^{-2}}{\left[\frac{1}{2} \pi^{2}+k_{Z}^{2} L^{2}\right]^{3}}
$$


Maximizing this over $k_{Z}$, we find that the optimum wavevector is

$$
k_{Z} L=k_{z}=\pi / 2=1.57 \text {. }
$$

This is close to the numerically determined value of 1.6 given in Table 1 . The maximum growth factor and time at which this maximum is attained are then

$$
\begin{gathered}
G_{\text {max }}(R)=0.82 \times 10^{-3} R^{2}, \\
|2 A| t_{\text {max }}(R)=0.13 R .
\end{gathered}
$$

These relations are shown as dashed lines in Figure 3. We see that the scaling with $R$ agrees well with the numerical results, and the coefficient is also reasonably close.

\subsubsection{Constant Specific Angular Momentum Flow}

In this section we consider a rotating flow with $q=2$. The vorticity frequency $2 B$ vanishes, and so the term proportional to it is not present in equation (34). We can then write down the following plane wave solution

$$
\begin{gathered}
u=\frac{k_{Z}^{2}}{k_{X}^{2}+k_{Z}^{2}} 2 \Omega t v_{0} \exp \left[i k_{X} X+i k_{Z} Z-\nu\left(k_{X}^{2}+k_{Z}^{2}\right) t\right] \\
v=v_{0} \exp \left[i k_{X} X+i k_{Z} Z-\nu\left(k_{X}^{2}+k_{Z}^{2}\right) t\right] \\
w=-\frac{k_{X} k_{Z}}{k_{X}^{2}+k_{Z}^{2}} 2 \Omega t v_{0} \exp \left[i k_{X} X+i k_{Z} Z-\nu\left(k_{X}^{2}+k_{Z}^{2}\right) t\right] \\
\hat{p}=-\frac{i k_{X}}{k_{X}^{2}+k_{Z}^{2}} 2 \Omega v_{0} \exp \left[i k_{X} X+i k_{Z} Z-\nu\left(k_{X}^{2}+k_{Z}^{2}\right) t\right]
\end{gathered}
$$

where $v_{0}$ is an arbitrary amplitude.

This solution looks quite different from that for plane Couette flow. For instance, here $u$ and $w$ grow linearly with time at early time and $v$ remains constant, which is the reverse of the case for plane Couette flow. Also, now we have a non-zero pressure perturbation. Nevertheless, the energy growth factor has the same dependence on time as for plane Couette flow:

$$
G(t)=\frac{u^{2}(t)+v^{2}(t)+w^{2}(t)}{u^{2}(0)+v^{2}(0)+w^{2}(0)}=\left[1+\frac{k_{Z}^{2}}{k_{X}^{2}+k_{Z}^{2}}(2 \Omega t)^{2}\right] \exp \left[-2 \nu\left(k_{X}^{2}+k_{Z}^{2}\right) t\right] .
$$

Note that, for $q=2,2 \Omega=2 A$, so the result is in fact identical. The reason for this

close similarity is apparent when one considers the original dynamical equations (33)-(35). 
The only difference between plane Couette flow and $q=2$ flow is that for the former the term $2 \Omega v$ is missing in equation (33) and the term $-2 B u$ present and is equal to $-2 A u$ in equation (34), whereas for the latter the term $2 \Omega v$ is present and is equal to $2 A v$ in equation (33) and the term $-2 B u$ is missing in equation (34). The equations are thus very symmetrical, except that $X$ and $Y$ are interchanged in the two cases. The resulting flows look very different because of the switch in coordinates, but the growth is identical.

The rest of the analysis proceeds exactly as in the previous subsection. As before, we conclude that the optimum $k_{Z} L=k_{z} \sim 1.57$, that the maximum growth factor is $G_{\text {max }} \sim 0.82 \times 10^{-3} R^{2}$, and that the maximum growth happens at a time $t_{\text {max }} \sim 0.13 R$. As we saw in $\S 3$, the numerical results are indeed very similar for plane Couette flow and $q=2$ flow (Figure 3). The present analysis explains why this happens even though the dynamics are quite different.

\subsection{3. $q<2$ Flow}

Now we consider a more general flow with $q<2$. Such a flow has an angular momentum gradient that is stable according to the Rayleigh criterion. This leads to epicyclic oscillations with frequency $\kappa$ [see Eq. (31)].

Let us ignore viscosity. Then, defining $k \equiv\left(k_{X}^{2}+k_{Z}^{2}\right)^{1 / 2}$, the plane wave solution with maximum growth is given by

$$
\begin{gathered}
u=\frac{k_{Z}}{k} \frac{2 \Omega}{\kappa} v_{0} \exp \left(i k_{X} X+i k_{Z} Z\right) \sin \left(\frac{k_{Z}}{k} \kappa t\right), \\
v=v_{0} \exp \left(i k_{X} X+i k_{Z} Z\right) \cos \left(\frac{k_{Z}}{k} \kappa t\right), \\
w=-\frac{k_{X}}{k} \frac{2 \Omega}{\kappa} v_{0} \exp \left(i k_{X} X+i k_{Z} Z\right) \sin \left(\frac{k_{Z}}{k} \kappa t\right), \\
\hat{p}=-\frac{i k_{X}}{k^{2}} 2 \Omega v_{0} \exp \left(i k_{X} X+i k_{Z} Z\right) \cos \left(\frac{k_{Z}}{k} \kappa t\right) .
\end{gathered}
$$

The energy growth as a function of time is given by

$$
G(t)=\cos ^{2}\left(\frac{k_{Z}}{k} \kappa t\right)+\frac{2}{(2-q)} \sin ^{2}\left(\frac{k_{Z}}{k} \kappa t\right) .
$$

Clearly, the maximum possible growth is

$$
G_{\max }=\frac{2}{2-q}
$$


and the growth happens after a time proportional to one quarter the epicyclic period,

$$
t_{\max }=\frac{\pi}{2 \kappa}\left(\frac{k}{k_{Z}}\right)
$$

In the actual flow with walls and viscosity the growth will be a little less, as confirmed by the numerical results in Table 1 , but equation (62) gives a rigorous upper limit to the growth.

The key point of this analysis is that, for $q<2$, there is a limit to the growth that arises just from dynamics; specifically, it is caused by the presence of a non-vanishing epicyclic frequency. Moreover, the limiting growth in energy is just a factor of 4 for a Keplerian flow. The limit has nothing to do with viscosity. In contrast, both plane Couette flow and $q=2$ flow can have infinite growth as far as the dynamics are concerned and the limit to growth arises only from viscosity.

Balbus, Hawley \& Stone (1996) and Hawley, Balbus \& Winters (1999) suggested that the existence of epicyclic motion lends dynamical stability to flows with $q<2$ and that this makes these flows more resistant to turbulence. Our analysis confirms their suggestion for perturbations with vertical structure. However, their argument does not apply to the two-dimensional perturbations we consider next.

\subsection{Two-Dimensional Perturbations}

The last subsection explained why perturbations with vertical structure have very little growth for $q<2$. The growth is especially insignificant for a Keplerian flow. $\S 3$ showed that these flows have more growth for perturbations with non-zero $k_{Y}$. In fact, for a Keplerian flow, the maximum growth is for $k_{Z}=0, k_{Y} \neq 0$. We now consider such perturbations.

We consider a plane wave that is frozen into the fluid and is sheared along with the background flow (see Figs. 7, 8). If the flow starts at time $t=0$ with initial wave-vector $\left(k_{X i}, k_{Y}\right)$ in the $X Y$-plane, then the $X$-wavevector at later times is given by

$$
k_{X}(t)=k_{X i}+q \Omega k_{Y} t
$$

With the above definition of $k_{X}$, we consider a plane wave solution of the form

$$
u=u(t) \exp \left(i k_{X} X+i k_{Y} Y\right), \text { etc.. }
$$

Because of the non-zero $k_{Y}$, the Lagrangian time derivative is given by

$$
\frac{d}{d t}=\frac{\partial}{\partial t}-i q \Omega k_{Y} X
$$


The relevant plane wave solution in the absence of viscosity has been written down by a number of authors (e.g., Chagelishvili et al. 2003; Umurhan \& Regev 2004). Generalizing the solution for finite viscosity, we have

$$
\begin{gathered}
u=\zeta \frac{k_{Y}}{k^{2}} \exp \left(i k_{X} X+i k_{Y} Y-\nu \int_{0}^{t} k^{2}\left(t^{\prime}\right) d t^{\prime}\right) \\
v=-\zeta \frac{k_{X}}{k^{2}} \exp \left(i k_{X} X+i k_{Y} Y-\nu \int_{0}^{t} k^{2}\left(t^{\prime}\right) d t^{\prime}\right), \\
w=0 \\
\hat{p}=i \zeta\left(\frac{1}{k^{2}} 2 \Omega-\frac{k_{Y}^{2}}{k^{4}} 2 q \Omega\right) \exp \left(i k_{X} X+i k_{Y} Y-\nu \int_{0}^{t} k^{2}\left(t^{\prime}\right) d t^{\prime}\right),
\end{gathered}
$$

where $\zeta$ is the amplitude of the vorticity perturbation. Since $w=0$, we see that the perturbations are two-dimensional (hence the name "two-dimensional perturbations"). Also, the velocity components are independent of $q$ and it is the pressure that adjusts so as to keep the dynamics the same for all values of $q$. In fact, the above solution is valid even for plane Couette flow, provided we make the replacements $2 \Omega \rightarrow 0$ and $2 q \Omega \rightarrow-2 A$.

In the absence of viscosity, the energy growth is given by

$$
G(t)=\frac{k_{X i}^{2}+k_{Y}^{2}}{k_{X}^{2}+k_{Y}^{2}}
$$

that is, the energy is inversely proportional to the square of the total wave-vector $k^{2}=k_{X}^{2}+k_{Y}^{2}$. This result is easy to understand. For inviscid incompressible twodimensional flow, the vorticity $\nabla \times \vec{\vartheta}$ is exactly conserved. This means that $k \vartheta$ is constant, so the velocity scales inversely as $k$. The energy must then vary as $k^{-2}$. The energy is thus largest when $k$ is smallest. Using equation (64) we now see what is required if we wish to obtain a large energy growth. We need to start with a large negative value for $k_{X i}$. As time goes on, $k_{X}$ will become progressively less negative; as a result, $k$ will decrease and $G$ will increase. The maximum growth will be achieved when $k_{X}=0$, giving

$$
G_{\max }=1+\frac{k_{X i}^{2}}{k_{Y}^{2}}, \quad|2 A| t_{\max }=\frac{k_{X i}}{k_{Y}} .
$$

Now consider the effect of having rigid walls at $X= \pm L$. By the uncertainty principle, $k_{X}$ cannot become exactly zero, but must have a minimum magnitude, $k_{X, \min } \sim \pi / L$. The maximum energy growth is then approximately given by

$$
G_{\text {max }}\left(k_{X i}, k_{Y}, R=0\right)=\frac{k_{X i}^{2}+k_{Y}^{2}}{k_{X, \min }^{2}+k_{Y}^{2}} \sim \frac{k_{X i}^{2}}{(\pi / L)^{2}+k_{Y}^{2}},
$$


where we have assumed that $k_{X i} \gg k_{Y}$. Including also the effect of viscosity this becomes

$$
G_{\text {max }}\left(k_{X i}, k_{Y}, R\right) \sim \frac{k_{X i}^{2} L^{2}}{\pi^{2}+k_{Y}^{2} L^{2}} \exp \left(-\frac{2}{3 R} \frac{k_{X i}^{3} L^{2}}{k_{Y}}\right) .
$$

Maximizing this with respect to $k_{X i}$ and $k_{Y}$, we obtain

$$
k_{Y} L=k_{y} \sim \frac{\pi}{\sqrt{2}}=2.2, \quad k_{X i} L=k_{x i} \sim 1.3 R^{1 / 3}
$$

The maximum growth and the time of maximum are then

$$
G_{\max }(R) \sim 0.059 R^{2 / 3}, \quad|2 A| t_{\max }(R) \sim 0.59 R^{1 / 3}
$$

While the scalings with $R$ are accurate and agree with the numerical results presented in $\S 3$, the coefficients are approximate since they depend on the assumed value of $k_{X, \min }$. If instead of taking $k_{X, \min } L=\pi$, we select $k_{X, \min } L=1$, we find $k_{Y} L=0.71$, $k_{X i} L=0.89 R^{1 / 3}, G_{\max }(R) \sim 0.27 R^{2 / 3},|2 A| t_{\max }(R) \sim 1.25 R^{1 / 3}$. The results in $\S 3$ lie between these two estimates. In fact, if we choose $k_{X, \min } L \sim 1.7$, then we obtain $k_{Y} L=1.2$, $G_{\text {max }}(R) \sim 0.13 R^{2 / 3},|2 A| t_{\max }(R) \sim 0.88 R^{1 / 3}$, which agree with the numerical result (Table $2)$.

Finally, we can carry out the analysis by keeping $k_{Y}$ fixed and optimizing only $k_{X i}$. We then find that $G_{\max }\left(k_{Y}, R\right)$ and $t_{\max }\left(k_{Y}, R\right)$ vary as

$$
\begin{gathered}
G_{\text {max }}\left(k_{Y}, R\right)=\frac{\left(k_{Y} L\right)^{2 / 3}}{\left(k_{X, \min } L\right)^{2}+\left(k_{Y} L\right)^{2}} \exp \left(-\frac{2}{3}\right) R^{2 / 3} \\
|2 A| t_{\max }\left(k_{Y}, R\right)=\left(k_{Y} L\right)^{-2 / 3} R^{1 / 3}
\end{gathered}
$$

We see that $G_{\max }$ varies as $\left(k_{Y} L\right)^{2 / 3}$ for small $k_{Y} L$ and as $\left(k_{Y} L\right)^{-4 / 3}$ for large $k_{Y} L$. The time of maximum scales as $\left(k_{Y} L\right)^{-2 / 3}$ for all $k_{Y} L$. The above analytical results are plotted as dotted lines in Figure 6 , assuming $k_{X, \min } L=1.7$ as derived above. We see that the agreement with the numerical results is very good except at very large $R$ where the calculations are not very accurate.

\section{Discussion and Conclusions}

We have demonstrated that significant transient growth of perturbations is possible in a Keplerian flow between walls (as shown by Yecko 2004). Although the system does not have any unstable eigenmodes, nevertheless, because of the non-normal nature of the eigenmodes a significant level of transient energy growth is possible for appropriate 
choice of initial conditions. If the maximum growth exceeds the threshold for inducing turbulence, it is plausible that this mechanism could drive the system to a turbulent state. Presumably, once the system becomes turbulent it can remain turbulent as a result of nonlinear interactions and feedback among the perturbations.

In this so-called bypass mechanism for transition to turbulence, the maximum energy growth and the time needed for this growth are likely to be the main factors that control the transition to hydrodynamic turbulence. It has been observed in laboratory experiments that plane Couette flow can be made turbulent for Reynolds numbers above a critical value $R_{c} \sim 350$. According to our analysis, for $R=350$, the maximum energy growth is $G_{\max }(R=350)=145$, and the maximum occurs at time $t_{\max }=42.3$ (Fig. 3 ). Since a constant angular momentum disk $(q=2)$ behaves very similarly to plane Couette flow, the critical Reynolds number for turbulence for this case is also likely to be $R_{c} \sim 350$. For this $R$, the growth factor is $G_{\max }(R=350)=143.5$ and the time-scale is $t_{\max }=48.3$.

It is true that the underlying equations as well as the maximally growing modes in plane Couette flow (and a $\mathrm{q}=2$ disk) are different compared to a Keplerian flow, and so the turbulent phases in the two systems may have significant differences. However, the presence of similar boundary conditions may suggest similarities in the kinematic structure of the turbulence. In fact, a Keplerian disk and a constant angular momentum disk are two special cases of rotating shearing flows parameterized by $q$. Based on this, we make the plausible assumption that the threshold energy growth factor needed for transition to turbulence in a shear flow with any value of $q$ is $E_{c} \sim 145$. Applying this conjecture to the optimal two-dimensional perturbations of a Keplerian disk analysed in $\S 4.2$, we estimate the critical Reynolds number for a Keplerian flow to be $R_{c} \sim 3.4 \times 10^{4}$, i.e., a factor of 100 greater than for plane Couette flow. The time to reach the maximum is $t_{\max }=28.3$, which is comparable to that in plane Couette flow, and is not too large compared to the accretion time scale of a geometrically thin disk.

Instead of taking $R_{c} \sim 350$, which is perhaps somewhat optimistic since plane Couette flow needs to be perturbed significantly before it will become turbulent at this Reynolds number, we might wish to be conservative and assume $R_{c} \sim 1000$ for this flow. At this value of $R$, plane Couette flow and $q=2$ flow have $G_{\max }(R=1000) \sim 1200$ and $t_{\text {max }} \sim 120-140$. Applying the requirement $E_{c} \sim 1200$ to Keplerian flow, we find $R_{c} \sim 10^{6}$ and $t_{\text {max }} \sim 100$. Now the critical Reynolds number is a factor of 1000 greater than for plane Couette flow.

Why is the critical Reynolds number so much larger for a Keplerian disk compared to a constant angular momentum disk or plane Couette flow? The numerical results in $\S 3$ and the analytical work in $\S 4$ provide the answer, viz., the presence of epicyclic motions in a Keplerian disk. It is very interesting to note that the presence of epicyclic motion 
not only kills growth dramatically, it also changes the optimum wavevector $\left\{k_{y}, k_{z}\right\}$ of the perturbations needed to produce energy growth. For a constant angular momentum disk $(q=2)$ and plane Couette flow, both of which have zero epicyclic frequency, it is seen that growth is maximized for $k_{y} \sim 0$ (on the $k_{z}$ axis). Even for a very small shift in the value of $q$ below 2, corresponding to the introduction of a small epicyclic frequency, the location of maximum growth immediately moves significantly in the $k_{y}-k_{z}$ plane from the $k_{z}$ axis (see Fig. 4 which corresponds to $R=2000$ ). With decreasing $q$, the epicyclic motion of the disk increases, and correspondingly the optimum value of $k_{y}$ for growth increases while the optimum $k_{z}$ decreases. When $q=1.5$, i.e., when the disk is exactly Keplerian, the growth is maximum for $k_{z}=0$ (on the $k_{y}$ axis). To the best of our knowledge, this change in the location of the maximum in the $k_{y}-k_{z}$ plane has not been commented upon prior to this work.

The change between $q=2$ and $q=1.5$ may be completely understood analytically, as we show in $\S 4$. The important point is that the vertical perturbations $\left(k_{y}=0\right)$ that cause the large observed growth in a $q=2$ disk require an absence of epicyclic motions. When the epicyclic frequency is zero, the velocity perturbation is able to grow linearly with time and the energy grows quadratically. The only limit to growth is provided by viscosity, which gives a scaling $G_{\max } \propto R^{2}$. However, once there is a non-zero epicyclic frequency, the growth is immediately limited. Even in the absence of viscosity, only a modest level of growth is possible. In fact, for a Keplerian flow, the maximum growth that one can obtain from vertical perturbations is only 4, well below the critical growth needed for turbulence. If vertical perturbations were the sole route to turbulence, then a Keplerian flow could never make the transition to turbulence.

However, as $\S 4.2$ shows, there are other kinds of perturbations, specifically twodimensional perturbations with $k_{z}=0$, which are not affected by epicyclic motions. For these perturbations, pressure fluctuations are able to absorb the effect of the Coriolis force. As a result, two-dimensional perturbations are able to grow to arbitrarily large values in the absence of viscosity. However, the growth is much reduced compared to the vertical perturbations described in the previous paragraph and it scales only as $R^{2 / 3}$. Thus, one needs much larger values of $R \sim 10^{4.5}-10^{6}$ before one can achieve the same level of energy growth as can be found in a $q=2$ disk for Reynolds numbers as small as $10^{2.5}-10^{3}$.

These results lead to a better understanding of the numerical simulations described in Balbus, Hawley \& Stone (1996) and Hawley, Balbus \& Winters (1999). Both papers showed that there is a close similarity between plane Couette flow and $q=2$ flow, in the sense that the two flows readily became turbulent in numerical simulations. However, once the authors reduced the value of $q$ below about 1.95, no turbulence was seen even when the 
flows were initialized with large perturbations. The authors suggested that the change in behavior is because of the dynamical stability imposed by the Coriolis force and epicyclic motions. Our analysis supports this conclusion.

However, Balbus, Hawley \& Stone (1996) and Hawley, Balbus \& Winters (1999) then proceeded to rule out the possibility of hydrodynamic turbulence in Keplerian disks. We do not agree with this conclusion. As we have shown, Keplerian disks can indeed support large transient energy growth, but they need much larger Reynolds numbers to achieve the same energy growth as plane Couette flow or $q=2$ flow. The numerical simulations probably had effective Reynolds numbers $\lesssim 10^{4}$ (because of numerical viscosity) which is below our most optimistic estimate of the critical Reynolds number. Thus, we suspect the simulations did not have sufficient numerical resolution to permit turbulence. In fact, Longaretti (2002) already suspected that the non occurrence of turbulence in previous simulations may be just due to the choice of low Reynolds number.

Although the problem we analysed is shear flow between walls, the optimum growing perturbations that we find for the Keplerian case are very similar to those described by Chagelishvili et al. (2003) and Umurhan \& Regev (2004) for an infinite shear flow. The perturbations are basically plane waves that are frozen in the shearing flow. Initially, at $t=0$, the effective wave vector of the perturbation in the $x$ direction $\left(k_{x}\right)$ is negative, which means that we have very asymmetric leading waves. As time goes on, the wavefronts are straightened out by the shear and $\left|k_{x}\right|$ decreases. At the time when the growth is maximum, $k_{x} \sim 0$ (but not precisely 0 because of the walls, see $\S 4.2$ ) and the wavefronts become almost radial. At yet later time, the growth decreases and the wave becomes of a trailing pattern.

The above time evolution is very different from that seen for the optimum perturbations in plane Couette flow or in a $q=2$ disk. In plane Couette flow, the $x$-component of the perturbation, $u$ (i.e. the normal velocity), dominates over the other components, $v, w$, at $t=0$. However, $u$ remains at the same level for all time whereas $v$ and $w$ increase strongly up to the point of maximum growth before declining. The overall shape of the perturbation is roughly self-similar with time. For a constant angular momentum disk, on the other hand, it is $v$ which remains constant with time whereas $u$ and $w$ vary by large amounts. However, as in plane Couette flow, the solution is largely self-similar in character up to the maximum. Neither of these flows shows the shearing perturbations that are characteristic of the Keplerian problem (Figs. 7 and 8).

We conclude with an important caveat. While the demonstration of large energy growth is an important step, it does not prove that Keplerian disks will necessarily become hydrodynamically turbulent. Umurhan \& Regev (2004) have shown via two-dimensional simulations that chaotic motions can persist for a time much longer than the time scale 
$t_{\max }$ needed for linear growth. However, they also note that their perturbations must ultimately decline to zero in the presence of viscosity. To overcome this limitation, it is necessary to invoke three-dimensional effects. Secondary instabilities of various kinds, such as the elliptical instability, are widely discussed as a possible route to self-sustained turbulence in linearly perturbed shear flows (see the review article by Kerswell (2002); see also e.g. Hellberg \& Orszag 1988; Le Diześ \& Rossi 1996). It remains to be seen if these three-dimensional instabilities are present in perturbed flows such as those shown in Figures 7 and 8 . If they are, one will in addition have to show that they lead to non-linear feedback and 3-dimensional turbulence.

We would like to thank the referee for various suggestions that improved the presentation of the paper. This work was supported in part by NASA grant NAG5-10780 and NSF grant AST 0307433.

\section{A. Appendix: Method to Compute the Transient Growth}

To compute the optimum growth, first we need to evaluate the 2-norm of $Q$. From (29) it is clear that the 2-norm depends on $\mathcal{L}$ which consists of $\mathcal{L}_{o s}$ and $\mathcal{L}_{s q}$. The underlying Hilbert space of the second order linear operator, $\mathcal{L}_{s q}$, is $\mathcal{H}_{s q}=L^{2}[-1,1]^{5}$. Therefore the inner product of $\hat{\zeta}_{1}, \hat{\zeta}_{2} \in \mathcal{H}_{s q}$ is defined as

$$
\left(\hat{\zeta}_{1}, \hat{\zeta}_{2}\right)_{L}=\int_{-1}^{1} \hat{\zeta}_{2}^{*} \hat{\zeta}_{1} d x
$$

The domain of $\mathcal{L}_{s q}$, that is $\mathcal{D}_{s q}$, is the set of functions $\{\psi\}$ which have a second derivative in $L^{2}[-1,1]$ satisfying $\psi( \pm 1)=0$. Following DiPrima \& Habetler (1969) we also define the underlying Hilbert space of $\mathcal{L}_{o s}, \mathcal{H}_{o s}$, consisting of the set of functions $\{\psi\}$ having a second derivative in $L^{2}[-1,1]$ satisfying $\psi( \pm 1)=0$. Therefore, for $\hat{u}_{1}, \hat{u}_{2} \in \mathcal{H}_{o s}$ the inner product is defined as

$$
\left(\hat{u}_{1}, \hat{u}_{2}\right)_{H}=\left(D \hat{u}_{1}, D \hat{u}_{2}\right)_{L}+k^{2}\left(\hat{u}_{1}, \hat{u}_{2}\right)_{L}
$$

The domain of $\mathcal{L}_{\text {os }}$, that is $\mathcal{D}_{\text {os }}$, is the set of functions $\{\psi\}$ that have a fourth derivative in $L^{2}[-1,1]$ satisfying $\psi( \pm 1)=\psi^{\prime}( \pm 1)=0$. Therefore the underlying Hilbert space of $\mathcal{L}$ is $\mathcal{H}=\mathcal{H}_{o s} \times \mathcal{H}_{s q}$ and the corresponding domain is $\mathcal{D}=\mathcal{D}_{o s} \times \mathcal{D}_{s q}$. Thus combining (A1) and (A2) and with some algebra, the inner product for $Q_{1}, Q_{2} \in \mathcal{H}$ can be written as

$$
\left(Q_{1}, Q_{2}\right)=\left(\mathcal{F} \hat{u}_{1}, \hat{u}_{2}\right)_{L}+\left(\hat{\zeta}_{1}, \hat{\zeta}_{2}\right)_{L}
$$

\footnotetext{
${ }^{5}$ The Hilbert space is defined as a complete vector space with an inner product.
} 
where $\mathcal{F}=-\left(D^{2}-k^{2}\right)$.

Now following Butler \& Farrell (1992) the perturbation energy density can be evaluated as

$$
E=\frac{1}{2 V} \int_{-1}^{1} \int_{0}^{a} \int_{0}^{b}\left(u^{2}+v^{2}+w^{2}\right) d z d y d x
$$

where $a=2 \pi / k_{y}, b=2 \pi / k_{z}$, and $V=2 a b$ is the integration volume. The physical velocity components are the real quantity obtained as

$$
u=\frac{1}{2}\left\{\hat{u} \exp \left[i \vec{k} \cdot \vec{r}_{p}\right]+\hat{u}^{*} \exp \left[-i \vec{k} \cdot \vec{r}_{p}\right]\right\}
$$

Now replacing $\left(v^{2}+w^{2}\right)$ in terms of $\zeta$ and $d u / d x$ in (A4) and integrating over $y$ and $z$ we obtain

$$
E=\frac{1}{8 k^{2}} \int_{-1}^{1}\left[k^{2} \hat{u}^{\dagger} \hat{u}+\frac{\partial \hat{u}^{\dagger}}{\partial x} \frac{\partial \hat{u}}{\partial x}+\hat{\zeta}^{\dagger} \hat{\zeta}\right] d x
$$

where $\hat{u}$ and $\hat{\zeta}$ are considered to be $N$ dimensional column matrices. Now combining (30), (A3) and (A6) we obtain

$$
8 k^{2} E=\left\|Q_{K}\right\|^{2}=C^{\dagger} e^{i \Sigma_{K} t} \hat{Q} e^{-i \Sigma_{K} t} C,
$$

where $\hat{Q}$ is a $K \times K$ Hermitian matrix whose $i j$ th element is the inner product of $\tilde{Q}_{i}$ and $\tilde{Q}_{j}$,

$$
\hat{Q}_{i j}=\left(\tilde{Q}_{i}, \tilde{Q}_{j}\right)=\left(\mathcal{F} \tilde{u}_{i}, \tilde{u}_{j}\right)_{L}+\left(\tilde{\zeta}_{i}, \tilde{\zeta}_{j}\right)_{L}
$$

Decomposing $\hat{Q}$ in terms of a matrix $W$ according to $\hat{Q}=W^{\dagger} W$, and combining (29) and (A7) , we obtain the expression for the optimum growth (see also Reddy \& Henningson 1993 and Schmid \& Henningson 1994),

$$
G_{K}(t)=\operatorname{maximum}\left(\frac{\left\|W \exp \left[-i \Sigma_{K} t\right] C\right\|_{2}^{2}}{\|W C\|_{2}^{2}}\right)=\left\|W \exp \left[-i \Sigma_{K} t\right] W^{-1}\right\|_{2}^{2},
$$

where the subscript 2 denotes the 2-norm or Euclidian norm. The 2-norm of the matrix $W \exp \left[-i \Sigma_{K} i\right] W^{-1}$ can be evaluated by means of a singular value decomposition. Then, for a given $t$, the square of the highest singular value is the maximum energy growth, $G_{\max }(t)$, for that time. $W$ can be computed easily by a similarity transformation of $\hat{Q}$

$$
\hat{Q}=S \sqrt{\hat{Q}_{d}} S^{\dagger} S \sqrt{\hat{Q}_{d}} S^{\dagger}=W^{\dagger} W
$$


where $S$ is a unitary matrix and $\hat{Q}_{d}$ is a diagonal matrix consisting of the eigenvalues of $\hat{Q}$ along the diagonal. Therefore one only needs to construct the matrix $\hat{Q}$ to compute $G_{K}(t)$, while $\Sigma_{K}$ is immediately available from the eigenvalues of $\mathcal{L}$. From (A6), (A7) and (A8), in the finite-difference approximation $\hat{Q}$ can be written as

$$
\begin{aligned}
\hat{Q} & =\Delta x\left[k^{2} U^{\dagger} U+\frac{\partial U^{\dagger}}{\partial x} \frac{\partial U}{\partial x}+Z^{\dagger} Z\right], \\
U_{m j} & =\tilde{u}_{m j} ; \quad Z_{m j}=\tilde{\zeta}_{m j}
\end{aligned}
$$

where $m$ runs between 1 and $N$ (points on the finite-difference grid) and $j$ ranges from 1 to $K$ (mode numbers).

\section{REFERENCES}

Afshordi, N., Mukhopadhyay, B. \& Narayan, R. 2005, ApJ (submitted); AMN05.

Balbus, S. \& Hawley, J. 1991, ApJ, 376, 214.

Balbus, S., Hawley, J., \& Stone, J. 1996, ApJ, 467, 76.

Bech, K. \& Andersson, H. 1997, J. Fluid Mech., 347, 289.

Blaes, O. \& Balbus, S. 1994, ApJ, 421, 163.

Butler, K. \& Farrell, B. 1992, Phys. Fluids A, 4(8), 1637.

Chagelishvili, G., Zahn, J.-P., Tevzadze, A. \& Lominadze, J. 2003, A\&A, 402, 401.

Chandrasekhar, S. 1960, Proc. Natl. Acad. Sci., 46, 53.

DiPrima, R. C., \& Habetler, G. J. 1969, Arch. Rat. Mech. Anal., 34, 218.

Drazin, P. \& Reid, W. 1981, in Hydrodynamic Stability, Cambridge.

Farrell, B. 1988, Phys. Fluids, 31, 2093.

Fleming, T. P., Stone, J. M., \& Hawley, J. F. 2000, ApJ, 530, 464

Fromang, S., Terquem, C. \& Balbus, S. 2002, MNRAS, 329, 18.

Gammie, C. 1996, ApJ, 457, 355.

Gammie, C. \& Menou, K. 1998, ApJ, 492, L75. 
Goldreich, P. \& Lynden-Bell, D. 1965, MNRAS, 130, 125.

Goldreich, P. \& Tremaine, S. 1978, ApJ, 222, 850; 1979, ApJ, 233, 857.

Goodman, J. 2003, MNRAS, 339, 937.

Hawley, J., Balbus, S. \& Winters, W. 1999, ApJ, 518, 394.

Hawley, J., Gammie, C. \& Balbus, S. 1995, ApJ, 440, 742.

Hawley, J., Gammie, C. \& Balbus, S. 1996, ApJ, 464, 690.

Hellberg, C. \& Orszag, S. 1988, Phys. Fluids, 31(1), 6.

Ioannaou, P. \& Kakouris, A. 2001, ApJ, 550, 931.

Johnson, B., \& Gammie, C. 2005a, ApJ (submitted), astro-ph/0501005; 2005b, ApJ (submitted).

Landau, L., \& Lifshitz, E. 1989, in Fluid Mechanics, Second Edition, Oxford: ButterworthHeinemann.

Le Diześ, S., Rossi, M. \& Moffatt, K. 1996, Phys. Fluids, 8(8), 2084.

Longaretti, P. 2002, ApJ, 576, 587.

Lynden-Bell, D. \& Pringle, J. 1974, MNRAS, 168, 603.

Kerswell, R. 2002, Ann. Rev. Fluid Mech., 34, 83.

Menou, K. 2000, Science, 288 (5473), 2022.

Menou, K., \& Quataert, E. 2001, ApJ, 552, 204.

Narayan, R., Goldreich, P., \& Goodman, J. 1987, MNRAS, 228, 1.

Orr, W. 1907, Proc. R. Irish. Acad. A, 27, 9.

Orszag, S. 1971, J. Fluid Mech., 50, 689.

Reddy, S. \& Henningson, D. 1993, J. Fluids Mech., 252, 209.

Richard, D. \& Zahn, J.-P. 1999, A\&A, 347, 734.

Romanov, V. 1973, Funct. Anal. Appl., 7, 137.

Schmid, P., \& Henningson, D. 1994, J. Fluids Mech., 277, 197. 
Shakura, N., \& Sunyaev, R. 1973, A\&A, 24, 337.

Swinney, H. \& Gollub, J. 1981, in Hydrodynamic Instabilities and the Transition to Turbulence, Springer-Verlag.

Tevzadze, A., Chagelishvili, G., Zahn, J.-P., Chanishvili, R., \& Lominadze, J. 2003, A\&A, 407,779 .

Trefethen, L., Trefethen, A., Reddy, S. \& Driscoll, T. 1993, Science, 261, 578.

Umurhan, O., \& Regev, O. 2004, A\&A, 427, 855.

Velikhov, E. 1959, J. Exp. Theor. Phys. (USSR), 36, 1398.

Yecko, P. 2004, A\&A, 425, 385. 